\title{
Die Rumpfbewegung der Kunstbeinträger und ihr Zusammenhang mit der konstruktiven Ausbildung der Kunstbeine.
}

\author{
Von \\ Dr. $=$ Ing. Bruno Bloch-Berlin. \\ Mit 87 Abbildungen im Text. \\ (Eingegangen an 24. August 1913.)
}

\section{Die frïheren Untersnchungen der Ganghewegung. Die Rumpfbewegung des Gesnnden.}

Die früheren Untersuchungen ïber den menschlichen Gang beschäftigen sich durchweg mit demgesunden Mensohen. Schon frühsind nach dieser Richtung Untersuchungen ron den Brüdern $W$ eber ${ }^{1}$ ) angestellt worden, an die spitter Braune und Fischer.) anknïpten. Letztere haben in einer sehr interessanten. in ihrem Buche ausführlich beschriebenen Weise aus Photogrammen eines gehenden Manness rechnerisch die Bewegungen gewisser Punkte des Körpers beim Gehen in Beziehung auf ein dreiachsiges rechtwinkliges Koorcinatensystem ermittelt. Bei den Versuchen, die sich allerdings nur auf ein einziges Individuum erstreckten, wurden diesem an den Füßen. den Unter-, Oberschenkeln, Linter-, Oberarmen und am Kopf Geißlersche Röhren befestigt. die in einem dunklen Raum mit rasch aufeinander folgenden Unterbrechungen zum Aufleuchten gebracht wurden. Die Röhren wurden, während der Hiann ging, auf beiden Seiten mittels je zweier photographiseher Apparate, die in einem Winkel von $60^{\circ}$ zueinander standen, aufgenommen, so daß die Bilder die Lage der Röhren und somit auch der verschiedenen Körperteile in den aufeinander folgenden Phasen eines Schrittes zeigten. Aus diesen Photogrammen ließen sich durch Messungen die Koordinaten der Fußspitze, der Wittelpunkte der Fuß-, Knie-, Hüft-, Hand-, Ellbogen- und Schultergelenke sowie des Kopf-

1) MEchanik der menschlichen Gehwerkzeuge. Eine anatomisch-physiologische Cntersuchung von den Brüdern Wilhel $\mathrm{m}$ Weber und Eduard Weber, Göttingen 18.36. Neu herausgegeben in Wilhelm Webers gesammelten Werken, Band VI.

2) Willelm Braune und Otto Fischer, Der Gang des Menschen, I. Teil, Leipzig 1895, und Otto Fischer, Der Gang des Menschen, II. Teil, Lcipzig 1899. 
scheitelpunktes mit Bezug auf ein feststehendes, dreiachsiges Koordinatensystem feststellen und hieraus wiederum die Koordinaten der Mittelpunkte zwisehen den beiden Schultergelenken und den beiden Hüftrelenken.

Es zeigte sich dabei. daß bei dem normalen Gange des gesunden Hensehen die einzelnen Teike des Körpers. ror allem auch der für die vorliegende Intersuchung allein interessierende Rumpf, relativ zu einem mit dem Körper gleichmäß3ig rorwärts bewegt gedachten Koordinatensystem geschiossene Raumkurven beschreiben, die sich aus den berechneten Koordinaten leicht kon-

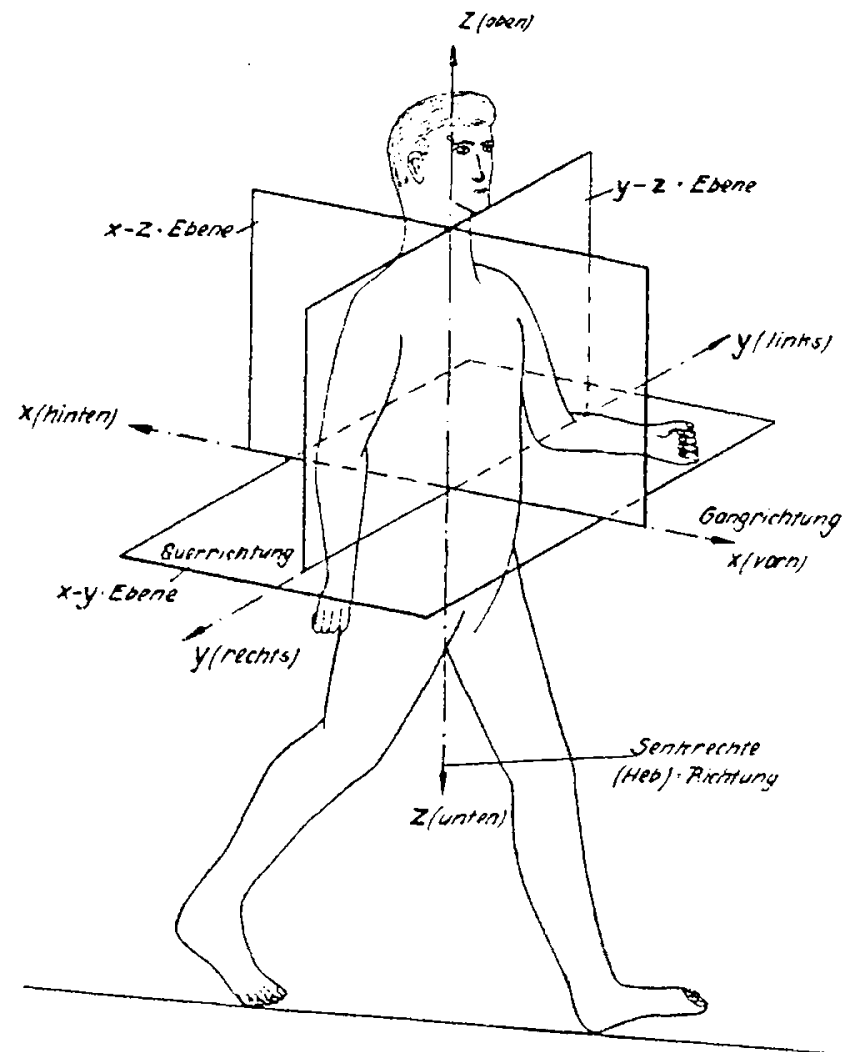

Abl. 1 .

struicren lassen. Die Bewegingen lassen sich am besten veranschaudichen. wenn man dic Ausdehnungen der Raumkurven in den drei Koordinatenrichtungen unibhängigr voneinander als Funktion der Zeit betrachtet. Die Knordinatenrichtungen wählt man mit Fischer am besten, wie aus $A b b .1$ ersichtlich, also so, daß die X-Richtung in die Gangrichtung fällt, die Y-Riehtung unter $90^{\circ}$ zur Gangrichtung in der wagerechten Ebene (Frontalebene) liegt und die Z-Richtung scnlirecht von unten nach oben geht.

Da hier nur die Bewegung des Rumpfes untersucht werden soll, genügt es, die Bewegungsdiagramme dor beiden Schultergelenke und Hüftgelenke zu betrachten, da durch diese dje Rumpfbewegung im wesentlichen bestimmt ist. 
Diese Kurven sind unter Benutzung der von Fischer ausgerechneten Koordinaten in den Abb. 2-4 aufgezeichnet.

Fischer hat fïr den gesunden Henschen folgende Rumpfbewegungen ermittelt, die beim Gehen zn der gleichmäbigen Vorwirtsbewegung hinzukommen:

\section{A. Parallelverschiebungen des Runptes.}

\section{In der X-Richtung (dangrichtung), zweckmätig als Wanderbewegnng des Rumpfes zu bezeichnen.}

Der Charalter dieser Bewegung läßt sich aus Abb. 2 erkennen, welche die Bewegungsdiagramme für die beiden Schultergelenke und die beiden Hüftgelenke enthïlt. Die Abszissen stellen die Zeit dar, und zwar ist die ganze Länge der Kurren gleich der Zeit eines Dopnelsehritts, während die Ordinaten den bjs zu dem betreffenden Zeitpunkte in der Gangrichtung zurückgelegten

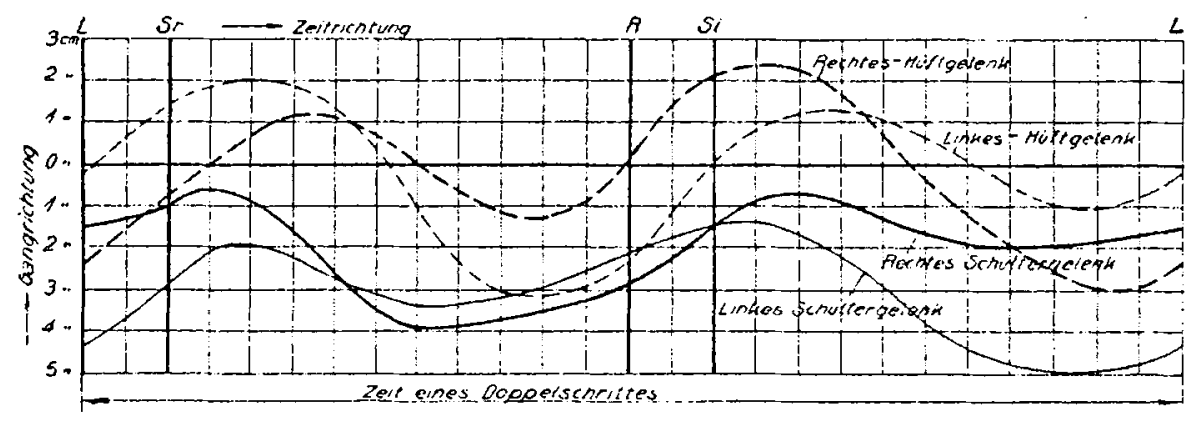

Abb. 2. Relative Bewegung der Schulter- und Hüftgelenke des Gesunden in der Gangrichtung ( $\mathrm{X}$-Richtung (Wanderbewegung).

Weg abzüglich des in der gleichen Zeit ron dem mit gleichbleibender Geschwindigkeit rorwizrts bewegten Koordinatensystem zurïckgelegten Weges angeben. Die ganze Zcit eines Doppelsehrittes ist in 26 Teile geteilt. Auf der Zeitachse sind die wichtigsten Phasen des Schrittes angemerkt, und zwar bedeutet

$$
\begin{aligned}
& \mathrm{R}=\text { Aufsetzen des rechten Beines } \\
& \mathrm{L}=\text { " "linken ", } \\
& \mathrm{S}_{\mathrm{r}}=\text { Beginn der Durchschwingung des rechten Beines } \\
& \mathrm{S}_{1}=" \text { " " } \quad \text { "Lnken " }
\end{aligned}
$$

Die O-Linie des Diagramms ist die wagerechte Projektion der Y-Z-Ebene. Die sich von der O-Linie nach oben erstreckenden Ordinaten bedeuten ein Vortreten und die nach unten gerichteten ein Zurückbleiben des betreffenden Gelenkpunktes gegenüber dem Koordinatensystem in der Gangrichtung zu den auf der O-Linie (X-Achse) angemerkten Zeiten.

Die vier Kurven zeigen einen wellenförmigen Verlauf, nach Art einer Sinuslinie, und zwar sind bei einem Doppelschritt zwei Maxima und zwei Minima vorhanden. $Z u$ den Zeiten $S_{1}$ und $S_{r}$, also beim Beginn der Schwingung der Beine, befindet sich der Rumpf in relativer Vorwärtsbervegung. Kurz nach- 
dem die Schwingung begonnen hat, hört die Vorwärtsbewegung anf, der Rumpf bewegt sich wieder relativ zu dem Koordinatensystem rückwirts und hat seine hinterste Stellnner erreicht. kurz bevor clas Aufsetzen des schwingenden Beines stattfindet. Die Kurve ist bezüglich des nach vorn und des nach linten gehenden Teiles nicht symmetrisch. vielmehr findet die Vorbewegung aus der äußersten Stellung hinten nach der außersten Stellung rorn in einer kürzeren Zeit statt, als die Bewegung von rorn nach hinten. Das Verhältnis ist etwa 2:3. Die Vorwärtsbewegung beginnt kurz vor dem Aufsetzen eines Beines, der größte und steilste Teil der Torbewegung liegt zwischen dem Aufsetzen eines Beines und dem Schwingungsbeginn des anderen Beines, also in der Zeit, wo beide Beine auf dem Borlen aufstehen.

\section{In der Y-Richtung (Frontalehene), zweckmäBig mit Selwenkhewegung zu bezeichnen.}

Dirse Bewegung ist in Abb. 3 gezeigt, in der die Kurven für die linke und rechte Seite, um sie besser gleichzeitig ïbersehen zu können, bei den Schulter-

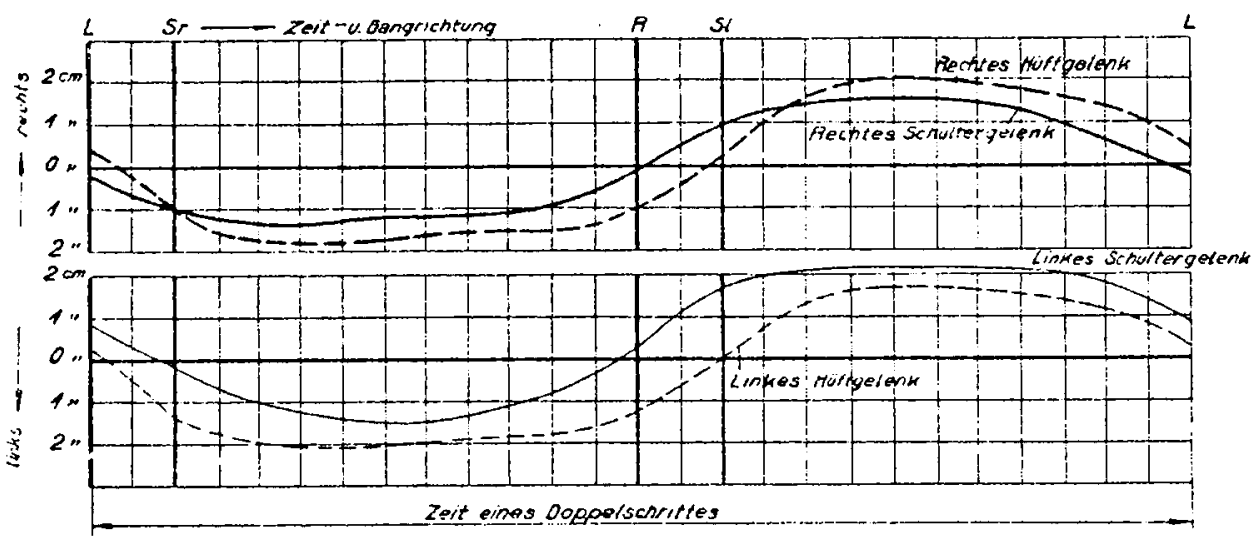

dbh. 3. Retative Bewegung der Schulter- und Hüftgelente des Gesunden in der Y.Richtung (Schwenktrewogring).

gelenken um $30 \mathrm{~cm}$ und bei den Hüftgelenken un $12 \mathrm{~cm}$ cinander genühert sind. Die O-Linien sind Projektionen der zur X-Z-Ebene parallelen, durch die mittlere Lage der betreffenden Gelenkmitten gehenden Ebenen; die Richtung nach oben bedeutet eine Bewegung nach rechts, die Richtung nach unten eine Betwegung nach links.

Den Kurven der vier Gelenke ist gemeinsam, daß sie während eines Doppelschrittes nur ein Maximum und ein Minimum haben, es findet also während eines Doppelschrittes nur eine einmalige Bewegung nach links und eine einmalige Bewegung nach rechts statt. Wenn das rechte Bein etwa die Hälfte der Sohwingbewegung ausgeführt hat, beginnt die Bewegung nach rechts und bei der entsprechenden Lage des linken Beines die Bewegung nach links; diese Punkte entsprechen also dem Maximum und Minimum. Wahrend der Zeit des Aufstehens beider Beine bewegen sich die Gelenke durch ihre Mittellage. Der aufund absteigende Teil der Kurven ist symmetrisch. 
3. In der Z-Richtung (Heben und Senken), zweckmäßig Hebbewegung genannt.

Die Diagramme dieser Bewegung sind in Abb. 4 dargestellt. Um die in senkrechter Pichtung weitauseinander liegenden Kursen besser mitcinander vergleichen zu können, sind in der Abbildung dit Kurven für die Hüftgelenke um $50 \mathrm{~cm}$ nach den Schultergelenken zu angehoben. Die Diagramme haben die Form von Sinus-Linien, die innerhalb eines Doppelschritts zwei Maxima und zwei Minima besitzen. Charakteristisch für die Kurven ist, daß das Minimum, also die tiefste Lage des Rumpfes, in beiden Fïllen zwischen den Jinien $R$ und $S_{1}$ einerseits und $L$ und $S_{r}$ andererseits erreicht wird, d. h. während beide

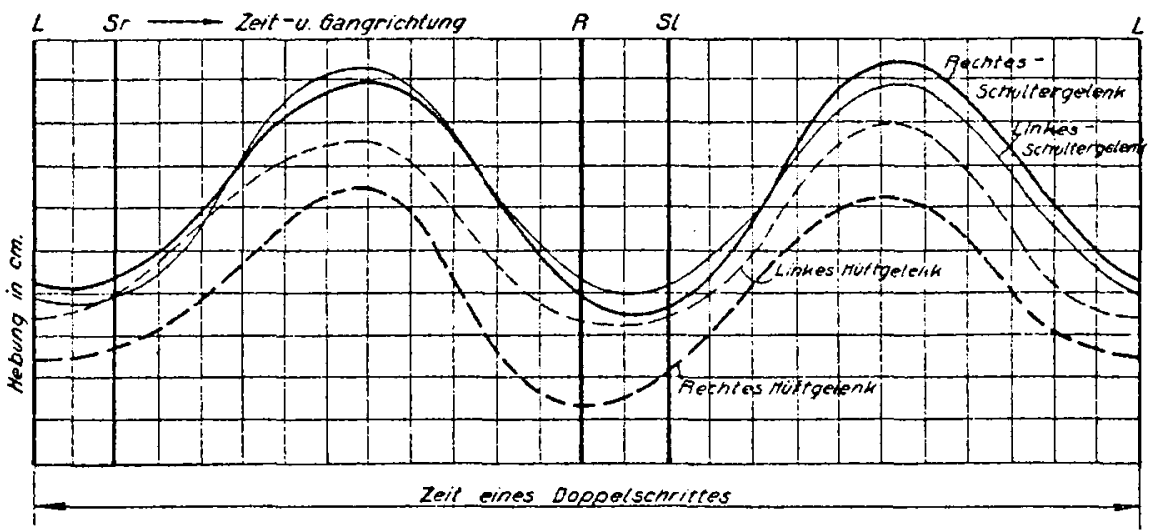

Abb. 4. Relative Bewegung der Schulter- und Hüftgelenke des Gesunden in der Z. Fichtung (Hebbewegung).

Beine aufstehen. Der Verlauf der Kurve ist im übrigen ein symmetrischer, der steigende und der abfallende Teil nehmen je etwa die Hialfte der Zeit in Anspruch. Das Maximum der Kurve (gröbte Hebung des Rumpfes) liegt gerade in der Mitte zwischen dem Beginn der Schwingung und dem Aufsetzen eines Beines, also etwa in dem Zeitpunkt. in dem das schwingende Bein durch die senkrechte Lage geht, dann senkt sich der Rumpf wieder und erreicht die niedrigste Lagge in dem Augenblick, in dem die beiden Beine in der Spreizstellung auf dern Fußboden aufstehen.

\section{B. Drehungen des Rumpfes.}

1. Drehung der Verbindungslinie der beiden Schultergelenke (Schulterlinie) und der Terhindungslinie der beiden Hiiftgelenke (Hüftlinie) um ihre Yittelpunkte in einer wagerechten Ebene.

Eine solehe Drehung lïßt sich daraus erkennen, daß in Abb. 2 die Kurven der beiden Schultergelenke ebenso wie die Kurven der beiden Hüftgelenke nicht zusammenfallen oder äquidistant sind. An ihren Schnittpunliten stehen Schulter- bzw. Hüftlinie senkrecht zur Gangebene, je weiter die Kurven voncinander abstehen, desto stärker sind diese Linien gegen die Gangebene (X-ZEbene) geneigt.

Es ergibt sich besonders deutlich für die Hüftlinie, daß sie während eines Doppelschrittes eine einmalige Schwingung nach Art einer Sinuslinie 
in der wagerechten Ebene ausführt. Vor dem Aufsetzen des rechten Beines etwa in dem Zeitpunkt, in dem der Rumpf am weitesten zurïcksteht, ist das rechte Hüftgelenk am weitesten nach rorn gedreht. Wiihrend der mun folgenden Vorwärtsbewegung des Rumpfes dreht sich gleichzeitig das linke Hüf gelenk vor; eine kurze Zeit nach dem Abschwingen des linken Beines erreicht die Hüftlinie die Normallage (senkrecht zur Gangebene), dann folgt eine wcitere Vordrehung des linken Hüftgelenkes, die kurz vor dem Iufsetzen des linken Beines wieder bei der weitesten Rïckwïrtslage des Rumpfes ihren größten Wert erreicht. Die nächste Nornallage wird nach dem Abschwingen des rechten Beines erreicht. Der gesamte Aussehlag der Hüftgelenke von der hintersten bis zur vordersten Drehlinge ist etwa $20 \mathrm{~mm}$.

Bei dem Schultergelenk zeigen die Kurven Unregehnäbigkeiten, dje offenbar auf ein unsymmetrisehes Ferhalten des Fersuch-Individumis zurückzuführen sind. Es ist aber zn erkennon. daß die Drehung der sohulterlinie ebenfalls nach einer Sinuslinie verliuft. Maximum und Minimum liegt an derselben Stelle wie bei der Drehung der Hüflinie, nur in umgekehrter Folge, d. h. bei weitester Vorwïrtalrehung des rechten Hüftgelenks ist das rechte Sohultergclenk am weitesten zurïckgedreht und umgekehrt. Der Ausschlag ist ein wenig geringer.

2. Drehung der Schulterlinie und der Hüftlinie um ihre Vittelpunkte in einer senkrechten Ebene.

Diese Drehung läßt sich in ähnlicher Weise aus Abb. 4 erkennen wie die Drehung in der wagerchten Ebene aus Abb. 2. Der Verlanf ist für die Sohulterlinie der Drehung der Schulterlinie in der wagerechten Ebene sehr ähnlich, nur ist der Ausschlag hier sehr klein, nicht iiber $5 \mathrm{~mm}$.

Die Bewegung der Hüftlinie in der senkrechten Ebene läßt bei einem Doppelschritt je drei Maxima und Minima erkennen; der Cresamtaussehlag ist ebenfalls nur gering, er betrïgt etwa $10 \mathrm{~mm}$.

3. Drehung der Verbindmngslinie von Schulterlinieumitte und Hüftlinienmitte (Rumpflinio) um die Hültlinienmitte als Drchpunkt, und zwar

a) ur eine senkrecht zur Gangrichtung liegencle Achse, also Neigung des Rumpfes nach rorn und hinten. Erkennhar ist diese Drehming aus Abb. 2; denkt man sich in dieser Abbildung die Kurven fiir die Bewegung der Schulter- und Hüftlinienmitten als arithmetische Nittel der Diagramme der betreffenden Gelenke eingezeichnet, so ergibt sich die Drehung der Pumpflinie aus den Differenzen der Ordinater dieser Kurven. In Abb. 5 sind dic Kurven der Schulter- und Hüftlinienmitten eingetragen, so da B sich die Rumpineigung ohne weiteres erkennen läßt. Sie wird durch eine Kurve dargestellt, die je zwei Haxima und Minima besitzt. Weiteste Vorwärtsneigung des Rumpfes findet kurz vor dem Aufsetzen eines Beines statt, weiteste Rückwartsneigung kurz vor der Mlitte des Schwingens eines Beines. Der Gesamtaussehlag ist $25 \mathrm{~mm}$.

b) um eine parallel zur Gangrichtung liegende Achse, also seitliche Leigung des Rumpfes. Diese Drehung ergibt sich entsprechend aus dem Verlauf der Kurren für die Schwenkbewegung der Hitten ron Schulter- wnd Hüftlinie 
gegeneinander. Die Bewegungen sind ähnlich denjenigen der Schulterlinie in der senkrechten Ebene. Gesamtausschligg $15 \mathrm{~mm}$.

Die von den Kunstbeintrügern ausgefiihrten Rumpfbewegungen sind bisher noch nicht untersucht worden. Zum ersten Wale sind wissenschaftliche Beobachtungen über den Gang ron Kunstbeintriigern von du Bois-Rey mon $\left(^{1}\right)$ angestellt worden. Diese Cntersuchungen beschränkten sich aber auf

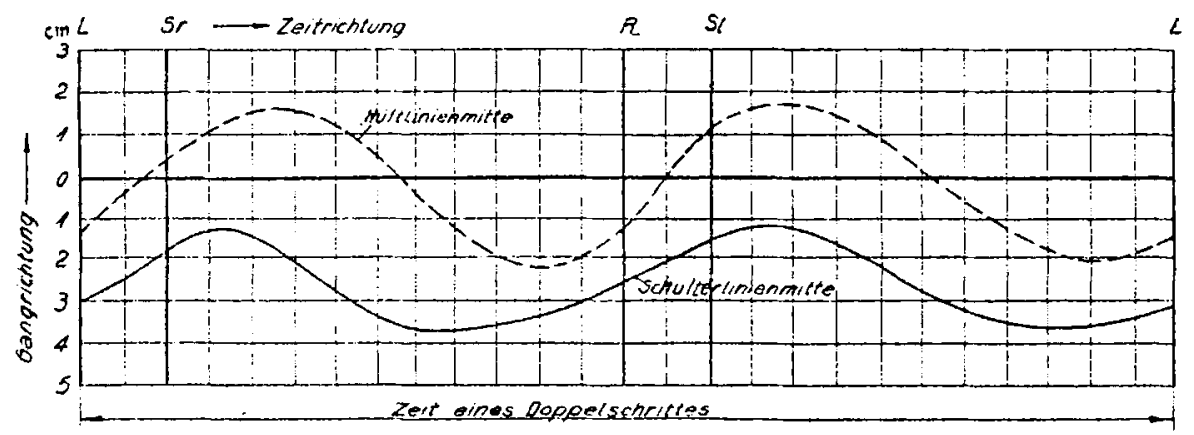

tbb. 5 . Wandrobeweyng der Schulter und Hiaftlinienmitte brim Gesunden.

die Feststellung der Beinbewegungen und bezweckten, aus der Verschedenheit der Beinbewegungen der untersuchten Kunstbeinträger ein Kriterium für. die Beurteilung der Güte ron Kunstbeinen zu gewinnen. Sie reichen aber für die Benteilung des Ganges mit Kunstbeinen nicht aus, weil sie ein sehr wesentliches Kenneeichen des Ganges, nämlich die. Rumpfbewegung: außer acht lassen.

\section{Die Bedentung der Rnupthewegnng des Gesunden fiir den Gang.}

Bevor mit der Vontersuchung der Rumpfbewegung des Kunstbeinträgers begonnen und ein Vergleich mit dem Gesunden durchgeführt werden kann, muß festgestellt werden, welche Bedeutung die im vorigen Abschnitt beschriebene Rumpfbewegung des Gesunden für den Gang hat, um einen Maßstab für den Fergleich mit der Rumpfbewegung des Amputierten zu gewinnen.

Bis zu den im vorigen Kapitel erwihnten Untersuehungen Fischers bestand ein Streit darüber, ob die Sohwingung des Beines beim Gehen eine reine, nur durch das Gewicht hervorgebrachte Pendelschwingung ist oder ob auch die Muskeln dabei mitwirken. Der Umstand, daB die Schwingung stets in der gleichen Weise erfolgt, auch wenn der Gehende nicht auf die Bewegung der Beine achtet. wurde zur Stützung beider Theorien herangezogen. Erst Fischer hat nachgewiesen, daß die Beinmuskulatur für die schwingbewegung eine sehr erhebliche Rolle spielt.

Um zu diesem Frgebnis zu kommen, denkt sich Fischer den Ober-bzw. Unterschenkel von dem äbrigen Teil des Körpers losgelöst und untersucht die auf die schwingenden Schenkel in jedem Augenblick einwirkenden äußeren

1) Über den Gang mit Kunstbeinen, R. du Bois-Rey mond, Zeitschrift des Vereins deutscher Ingenieure 1917, S. 645 . 
Kräfte. Wenn man zur Vereinfachung der Entersuchung die Schwingung als in einer Ebene erfolgend ansieht, also die nur geringe seitliche Schwankung vernachlässigt, so lassen sich diese Kräfte zusammensetzen zu einer im Sehwerpunkt des Schenkels angreifenden Kraft. Welche nach Größe und Richtung der Beschleunigung des Sehwerpunktes proportional ist und drei Drehmomenten, nämlich dem Drehmoment der Schwere, dom Drehmoment der Effektivkräfte und dem Drehmoment der Muskelkräfte. Die Summe dieser drei auf cine wagerechte durch den Schwerpunkt des Schenkels gelegte A hse bezogenen Drehmomente muß gleich sein dem Produkt aus der Winkelbeschleunigung des Schenkels in dem betreffenden Zeitpunkt bei seiner Drehung um den Sehwer punkt und dem auf die Drehachse bezogenen Trägheitsmoment.

Aus den aufgenommenen Photographien ließen sich nun alle Größen der genannten Gleichung turch Mlessung und Rechming finden, bis auf dis Drehmoment der Wuskelkräte, so dals man die Größe dieses Drehmomentes für jeden Zeitpunkt. der Schwingung aus der Gleichung berechnen konnte.

Die auf diese Weise ermittelten, durch die Muskeln auf den Oberschenkel während einer Schwingung ausgeübten Drehmomente sind in Abb. 6 graphisch als Funktion der Zeit dargestellt. Das Diagramm zeigt Mittelwerte aus den verschiedenen Fischerschen Versuchen. Die Werte oberhalb der O-Linie bedeuten Drehung entgegengesetzt der Lhrzeigerrichtung, die Werte unter der O-Linie Drehung in der Librzeigerrichtung.

Die Drehmomente nü̈ssen durch Kontraktion der an dem Oberschenkel angreifen-

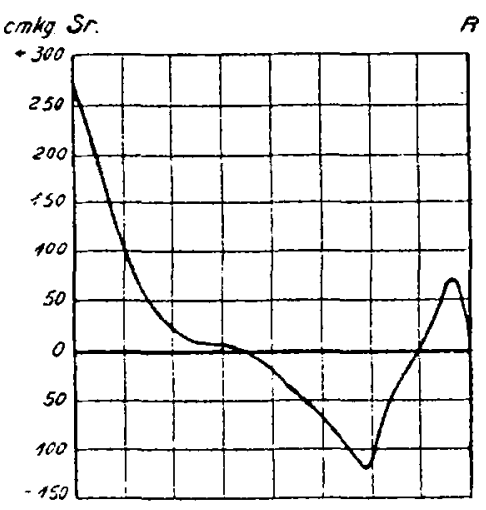

Ahb. (i). Drehmoutente der MLaskeln wärend der schwingung des Oberschenkels. den Muskeln oder durch Spannen cler Gelenkbïnder hervorgebracht werden, und damit steht wohl ein Teil der Relativbewegung der Hiift- und Schultergelenke regenüher dem gleichmäBig vorbewegten Koordinatensystem in Zusammenhang, wïhrend ein anderer Teil einfach den Zweek hat, das Gleichgewicht des Körpers aufrecht zu erhalten oder Paum für das durchschwingende Bein zu schaffen, un ein Anstoßen am Fußboden zu verhindern.

Für die drei Koordinatenrichtungen gilt im einzelnen folgendes:

\section{X-(Gang-) Richtung.}

Kurz vor dem Aufsetzen eines Beines, also wenn das andere Bein am weitesten nach hinten gestreckt ist, beginnt die zusätzliche Vorbewegung des Rumpfes, insbesondere der Hüften; der größte Teil der Vorbewegung überhaupt findet statt, während beide Beine aufstehen, so daB im Augenblick des $A b$ schwingens bei dem Schulter- und dem Hüftgelenk fast die vorderste Stellung erreicht ist. Wenn das Bein seine hinterste Streckstellung hat, sind die die Schwingung des Oberschenkels nach hinten begrenzenden Bänder des Hüftgelenks gespannt. Durch die während des Aufstehens beider Beine dann erfolgende starke Vorbewegung des Rumpfes wird diese Spannung der Bänder 
noch erhöht, und zwar in solchem Maße, daß die Bänder im Augenblick des Abschwingens den Oberschenkel mit großer Kraft vorschleudern. Hierdurch erklïrt sich wohl das sehr starke Drehmoment, mit dem die Mluskeln und Bïnder gerade bein Abschwingen des Beines (vgl. Abb. 6) wirken. Dieses Drehmoment nimmt bei der infolge des Schwingens rasch eintretenden Entspannung der Bänder sehr schnell ab, es erreicht die Null-Linie etwa in dem Zeitpunkte. in dem der Rumpf seine weiteste Lage nach vorn hat.

Inzwischen geht der Oberschenkel durch die senkrechte Lage hindurch und die Muskeln müssen, wie Abb. 6 zeigt, ein negatives (in der Uhrzeiger. richtung wirkendes) Drehmoment erzeugen. Es kommen hierbei die hinter dem Hüftgelenk vorbeigehenden Streckmuskeln des Oberschenkels in Tïtigkeit. Etwa gleichzeitig mit dieser Umkehrung der Richtung des Drehmomentes der Muskeln erfolgt auch die Cmkehrung der Relativbewegung der Schulterund Hüftgelenke, ein ursïchlicher Zusammenhang zwischen diesen beiden Vorgängen ist aber kaum vorhanden, vielmehr findet die Rückwärtsbewegung des Rumpfes wohl nur statt, damit kurz ror dem Aufsetzen des schwingenden Beines dif richtige Stellung erreicht wird. um in der oben angegebenen Weise durch erneute Torbewegung eine Spannung der Gelenkbünder des anderen uunmehr abschwingenden Beines vornehmen zu können.

Die Wanderberregung des Rumpfes scheint demnach in der Hauptsache den Zweok zu haben, durch Spannung der Hüftgelenksbünder und -muskeln das Abschwingen des Boines zu erleichtern.

Die von der Hüftlinie und der Schulterlinie ausgeführten Drehungen in der wagerechten Ebene sind wohl auf den Einflul 3 der an den betreffenden Gelenken angehängten Glieder zurückzuführen. Gerade beim Aufsetzen eines Beines hat die Hüftlinie eine solche Lage, daß das zu rlem aufgesetzten Bein gehörige Hüftgelenk am weitesten vorn ist. Die Drehung erfolgt also daclurch, daß das Standbein das zugehörige Hüftgelenk zurüchhält, während das schwingende Bein das zugehörige Hüftgelenk nach vorn zieht. Gleichzeitig dreht sich die Schulterlinie in umgekehrter Richtung, weil die Armschwingung stets in umgeliehrter Richtung erfolgt als die Beinschwingung derselben Körperseite.

Eine Folge cler Drehung der Hüftlinic ist eine Vergrößerung der Schrittlänge.

\section{Y-Richtuug.}

Da Arme, Beine und Kopf eine beachtenswerte, von der Rumpfbewegung unabhïngige seitliche Schwenkbewegung nicht ausführen, bedeutet die Schwenkbewegung des Rumpfes eine Verlegung des Gesamtschwerpunlites des Körpers nach rechts oder links um nahezu das gleiche Maß. Beim Aufstehen beider Beine ist die Mittellage vorhanden, beim Abschwingen eines Beines erfolgt die Schwenkung nach der entgegengesetzten Seite. Diese Verlegung des Schwerpunktes hat offenbar den Zweck, das abschwingende Bein zu entlasten und ein seitliches Umkippen des Körpers zu verhindern. Die Schwenkung braucht nur so groß zu sein, claß bei der in der Hauptsache durch die Schwerkraft erfolgenden Rückschwingung der Schwerpunkt gerade wieder die O-Linie schneidet, wemn das schwingende Bein aufgesetzt wird. Je nach der schwingungsdauer des Beines wird demnach der Ausschlag der Schwenkbewegung ein verschieden 
Die Rumpfbewegung der Kunstbeintrïger und ihr Zusammenhang usw.

großer sein. Hiermit deckt sich die Beobachtung, daßB die Schwenkbewegung um so kleiner wird, je rascher der Gang ist.

\section{Z-Richtung.}

Der leicht ein\%usehende Zweck der Hebbewegung des Rumpfes ist die Erleichterung des Durchschwingens der Beine. Der höchste Punkt der Diagramme (Abb. 4) liegt gerade in der Mitte der Schwingung der Beine, also in dem Zeitpunkte, in dem das schwingende Bein etwa durch die senkrechte Lage hindurchgeht und am meisten Gefahr besteht, daß der FuB gegen den Boden anschlïgt.

Auf die Drehung der Schulter- und Hüftlinie in der senkrechten Ebene sowie auf die Drehung der Rumpflinie soll, da diese Bewegungen nur klein sind, nicht näher eingegangen werden.

\section{Die Ermittlung der Rumpfbewegung der Kunstbein- trïger.}

Kunstbeintrüger führen beim Gehen, wie die Beobachtungen ergeben, ganz andersartige und erheblich größere Bewegungen insbesondere des Rumpfes wus als gesunde AIenschen. Diese Bewegungen können in einzelnen Fiillen auf besondere physiologische Einflüsse zurückzuführen sein, also z. B. auf eine Verlagerung gewisser Teile des Knochengerüstes als Folge der Amputation, cine ungewöhnliche Stumpfstellung (Abuluktionsstellung), zu geringe Länge oder Beweglichkeit des Stumpfes usw, im allgemeinen sind sie aber die Folge des Ersatzes des natürlichen Beines durch ein in der konstruktiven Gestaltung abweichendes Kunstbein und des damit verbundenen Fortfallens gewisser Muskeln. Infolge dieser physiologischen und mechanischen Einflüsse ist es für den Kunstbeinträger insbesondere bei Oberschenkelamputation - und nur von solchen Fällen soll in folgenden die Rede sein - vicht möglich, cinen dem normalen Gaing des gesunden Menschen gleichartigen Giung zu crzielen, es werden immer gewisse für den Kunstbeintrüger charakteristische Unterschiede vorhanden sein. Je schlechter die Konstruktion eines Kunstbeines ist, desto größer werden die Abweichungen des Canges der Kunstbeinträger von dem Gange des Gesunden sein. Es werden sich jedoch zwei Arten solcher Abweichungen feststellen lassen, nämlich solche, die durch die Amputation und das Tragen eines Kunstbeines äberhaupt und solche, die durch die Konstruktion des Kunstbeines bedingt sind. Je geringer die Abweichungen der letzterwähnten Art sind, je mehr also der charakteristische Gang des Kunstbeintrügers erreicht wird, als desto besser wird man unter Berücksichtigung etwa vorhandener besonderer physiologischer Einflüsse das Kunstbein zu bezeichnen haben.

Un die Rumpfbewegungen des Kunstbeinträgers und ihren Zusammenhang mit der lonstruktiven Gestaltung der Kunstbeine festzustellen, wurden mit einer Anzahl am Oberschenkel amputierter Soldaten mit verschiedener Stumpflänge und mit verschiedenen Beinsystemen photographische Gangaufnahmen gemacht. Da für die Zwecke dieser Arbeit der ron Fischer eingeschlagene Weg der Rechnung zil umständlich und zeitraubend war, wurden 
die Bewegungen aus den Photogrammen zeichnerisch ermittelt. Zn diesem Zwecke wurde eine zur tntersuchung von Armbewegungen bereits erprobte Apparatur benutzt, die dem Verfasser ron Herm Professor Dr.-Ing. SehJe. singer in der Prüfstelle für Frsatzglieder, Charlottenburg, zur Verfügung gestellt wurde.") Die Apparatur bestand aus kleinen, an den zu beobachtenden Körperteilen zu befestigenden Glühlampen, in deren Stromkreis ein Stimmgabelunterbrecher cingeschaltet war, so daß die Lampen in rascher Folge anfleuchteten und dunkel wurden. Die Aufnahme dieser Lampen bei der Bewegung des Körpers in einem verlunkelten Raume mußte eine fortlaufende, aus ein-

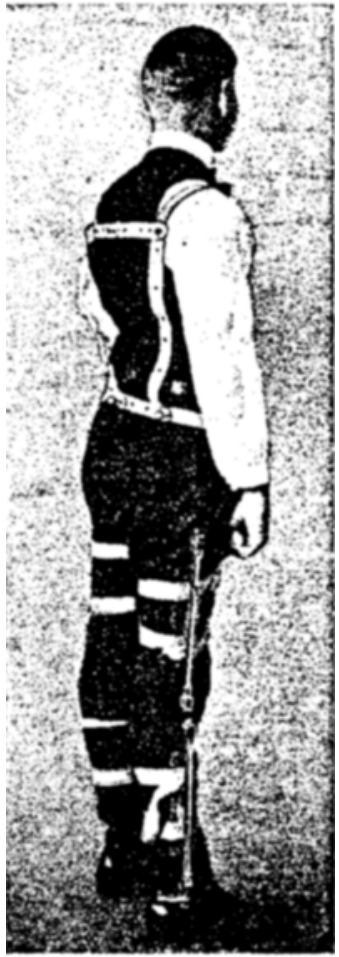

Abb. 7.

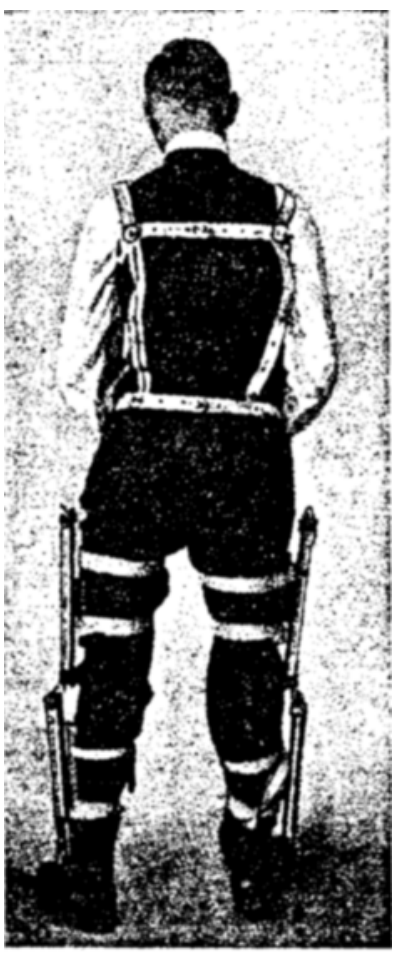

Abb. 8.

zelnen Punkten bestehende Kurve ergeben. Da es sich ledjglich um die Beobachtung der Rumpfbewegung handelte, wurden den Amputierten die Glühlampen an den beiden Hüftgelenken, den beiden Schultergelenken und den Mitten zwischen Schultergelenken und Hüftgelenken befestigt. Die Lampen wurden zu diescm Zreek auf Gurte aufgenäht, die um den Leib bzw. um die Achseln gelegt und mit Schnallen befestigt wurden (Abb. 7 und 8). Die so ausgerüsteten Leute wurden in einem dunblen Raume, während sie ohne Stock über ein Holzpodium, also auf glattem, ebenem Boden gingen, mit zwei gleichen photographischen Apparaten gleichzeitig von beiden Seiten aufgenommen.

1) Vgl. Mterkblatt Xir. 13 der Priifstelle für Ersatzglieder, Charlottenburg. 
Außerctem wurde eine Aufnahme von hinten mittels einer Stereoskopkamera gemacht.

Un auf den Bildern feststellen zu können, welche Punkte der Kurverı zu den verschiedenen Phasen der Beinbewegung gehören, wurden den aufzunehmenden Ieuten an beiden Ober- mol Unterschenkeln Geißlersthe Röhren angebunden, in gleicher Weise, we es von Fischer und von du Bois-Reymond bei ihren Untersuchungen gemacht wurde. Dic Geißlerschen Röhren, rlie ebenfalls mit Lnterbrechungen aufleuchteten, mußten unterhalb der Punktkurven Bilder der versehiedenen Stellungen der Beine während des Ganges ergeben

Vier solcher Aufnahnen sind in den Abb. 9--20 gezeigt. Die Aufnahmen Abb. 9-11 und Abb. 12-14 zeigen denselben Amputierten -- in Zukunft Amputierter I genannt - aber mit verschiedenen Kunstbeinen. Der Beintrïger war am rechten Oberschenkel ampntiert, stumpllänge 23 cm rom Damm gemessen, Körpergröße $176 \mathrm{~cm}$, Körpergewicht $60 \mathrm{~kg}$. Abb. 15-17 sind eine Aufnabme cines ebenfalls am rechten Oberschenkel Amputierten (Amputierter II) rnit einer Stumplänge ron $19 \mathrm{~cm}$, einer Körpergröße von $162 \mathrm{~cm}$ und einem Körpergewicht von $62 \mathrm{~kg}$. Bei beiden Ampntierten handelte s's sich um Leate, die das Kunstbein schon einige Zeit trugen und anerkannt gute Kunstbeine besaßen, jedoch hatte der Amputierte I schon eine grölere Cbung im Gehen erlangt, auch war sein Stumpf kräftiger.

Zum Vergleich mit diesen Anfnahmen ist in den Abb. 18 -.20 eine gleiche Gangaufnahme eines gesunden Hannes gezeigt, der eine Körpergröße von $178 \mathrm{~cm}$ und ein Körpergewicht von $82 \mathrm{~kg}$ hatte.

Der Stimmgabelunterbrecher arbeitete mit einer Cnterbrechungszahl ron $3 \tilde{5}$ pro Sekunde, während die Geißlersohen Röhren etwa 13 mal in der Sckunde aufleuchteten. Aus diesen Zahlen läßt sich die Dauer eines Schrittes und die Ganggeschwindigkeit feststellen, da ja die Länge eines Schrittes aus der Photographie ohne weiteres gemessen werden kann.

Aus den in der angegebenen Weise aufgenommenen Photogrammen tann man nun die Diagramme der Bewegungen des Rumpfes in den drei Koordinatenrichtungen durch folgende Ĺberlegung gewinnen:

\section{X-Richtung.}

Das Stereoskopbild zeigt zwar die vollständige Raumkurve, und man könnte schon aus ihr allein die gewünschten Resultate erzielen, aber nur durch sehr schwierige und umständliche photographische Meßmethoden. Bei einiger Ubung kann man angenäherte Werte aus den Stereoskopbildern auch dadurch crhalten, daß man sie in Stereoskop betrachtet und die Art und Größe der Bewegungen durch Vergleich mit den entsprechenden Aufnahmen des Gesunden abschätzt. Sehr schwierig, wenn nicht unmöglich ist diese Schätzung für die Bewegung des Rumpfes in der X-(Gang-)Richtung (Wanderbewegung) relativ zu dem mit gleichmäßiger Geschwindigkeit vorwärtsschreitenden Koordinatensystem, denn man müBte sich dazu in jedem Punkte der Kurve das bis dahin von dem System zurückgelegte Wegstück in Abzug gebracht denken. Man kann aber ein anschauliches Bild von der Wanderbewegung des Rumpfes aus den Seitenphotographien gewinnen, und zwar aus der Dichte der Punktfolge. Die 


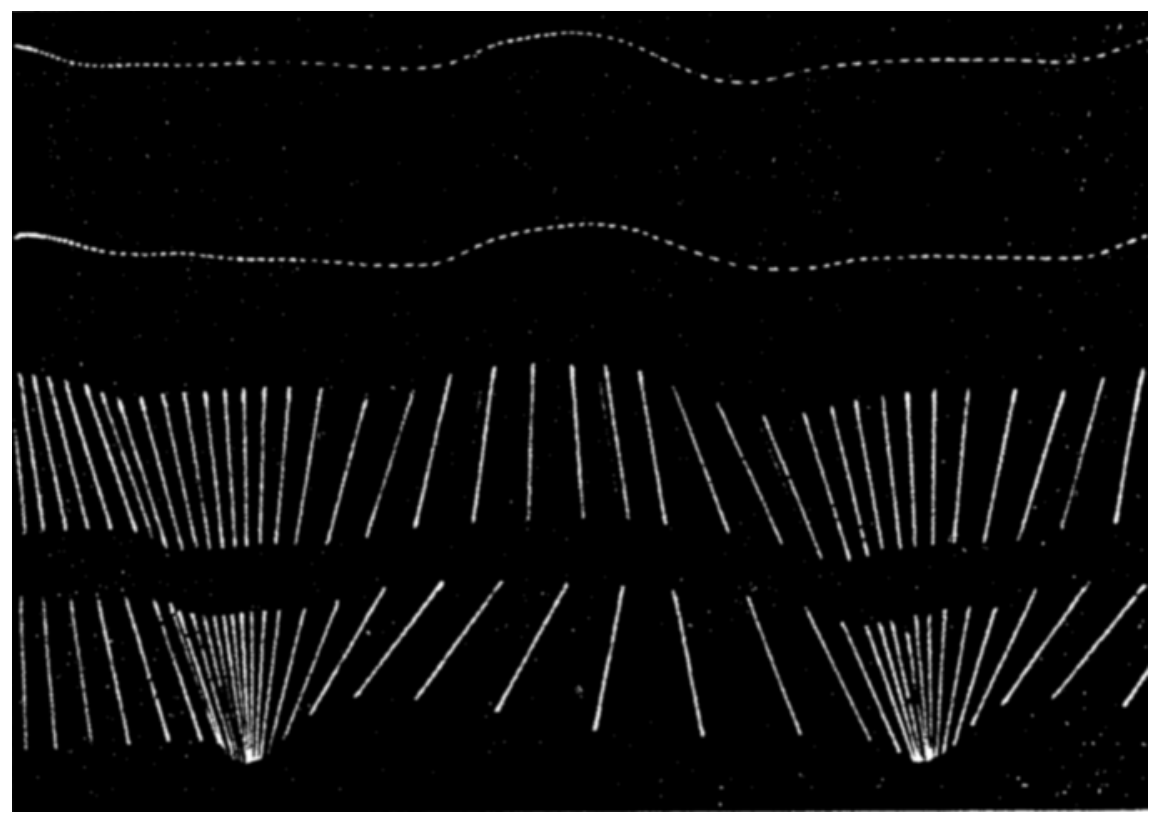

Abb. 9. Versuch Nr. 1. Aufnahme von rechts.

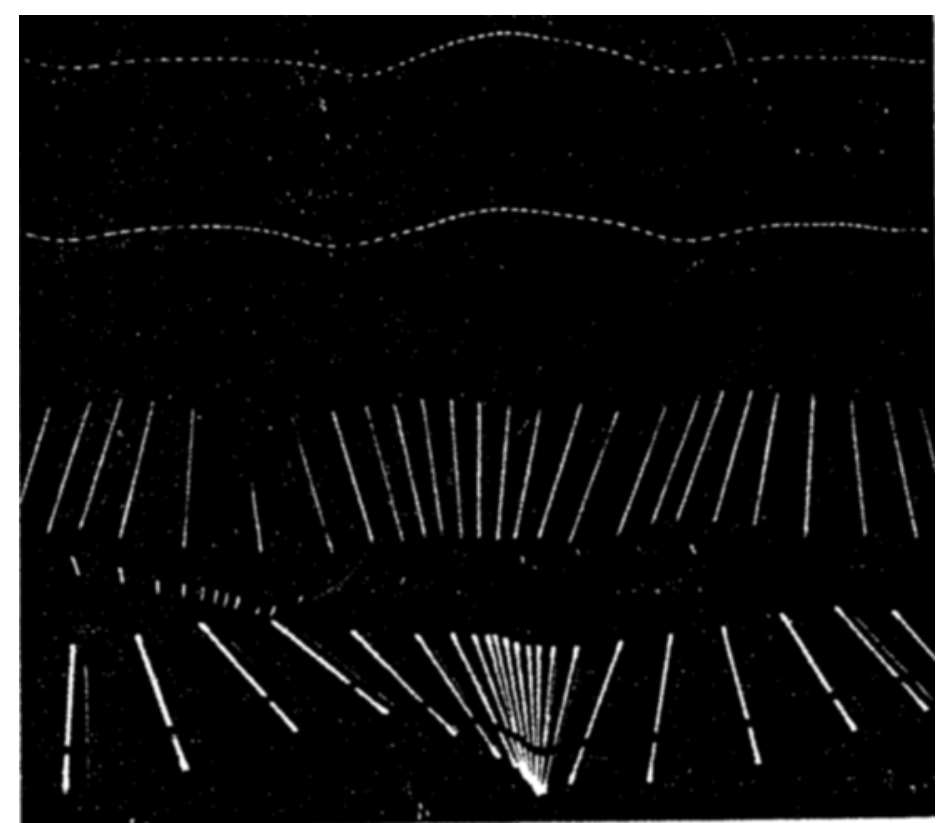

Abb. 10. Versucb Nr. 1. Aufnabme von links. 


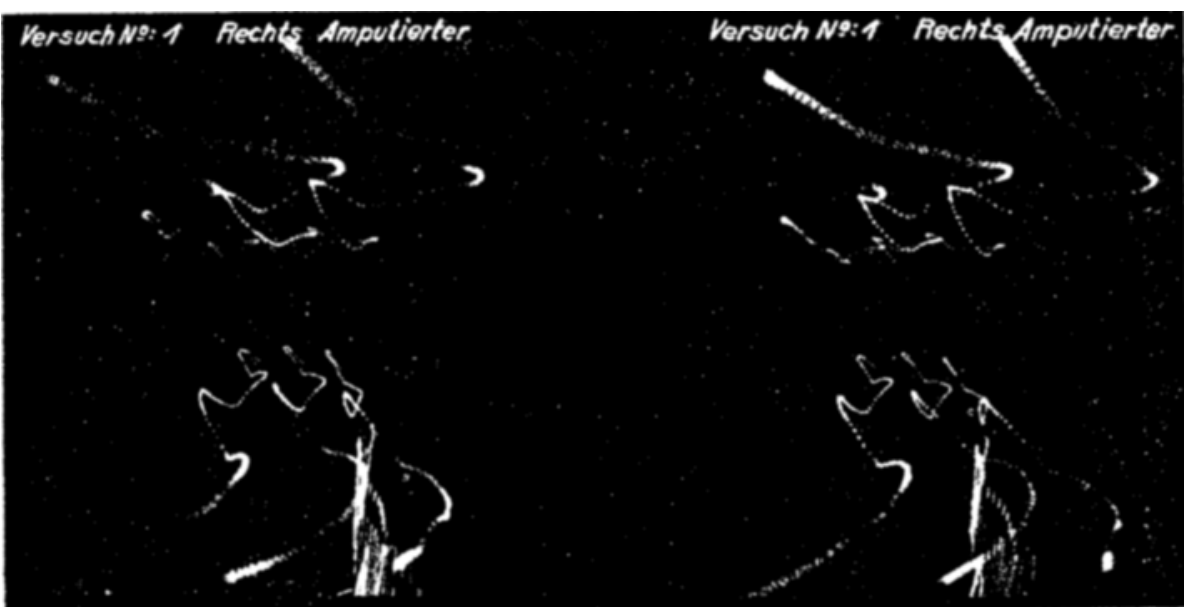

Abb. 11. Steroskopaufnahms van hinten.

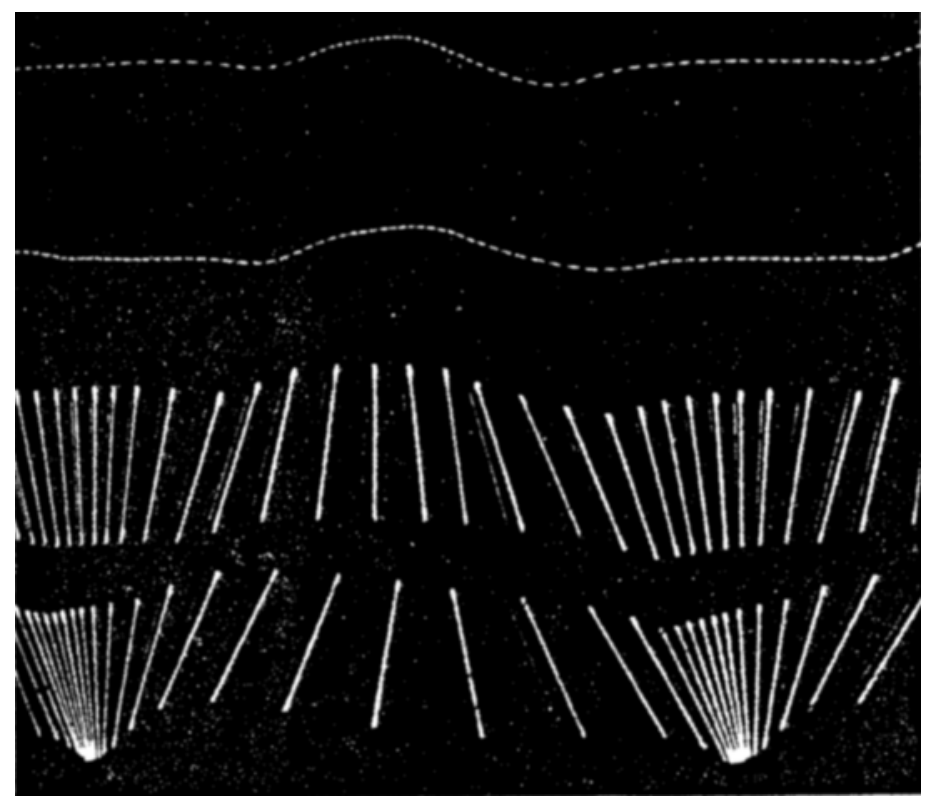

Ahb. 12: Feronch Nr. 2. tufnahum von reches. 


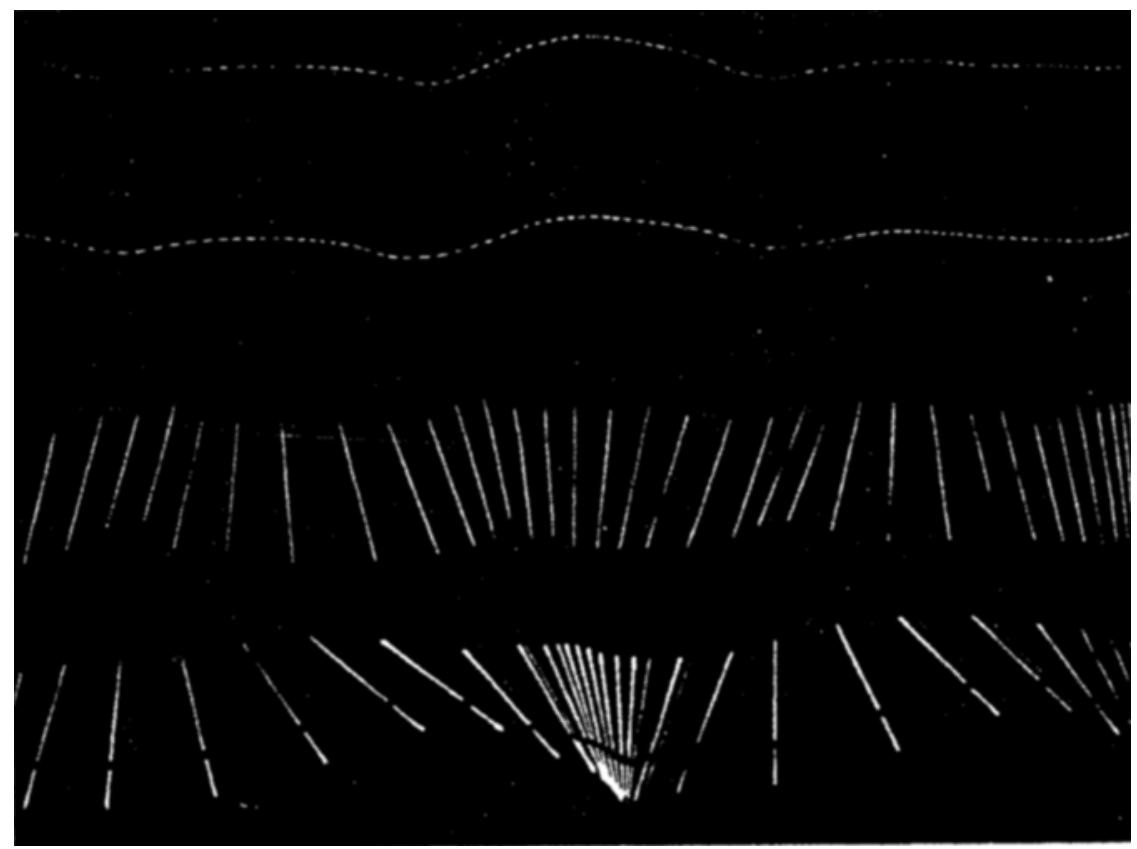

Abb. 13. Versuch Nr, 2. Aufnahme von links.

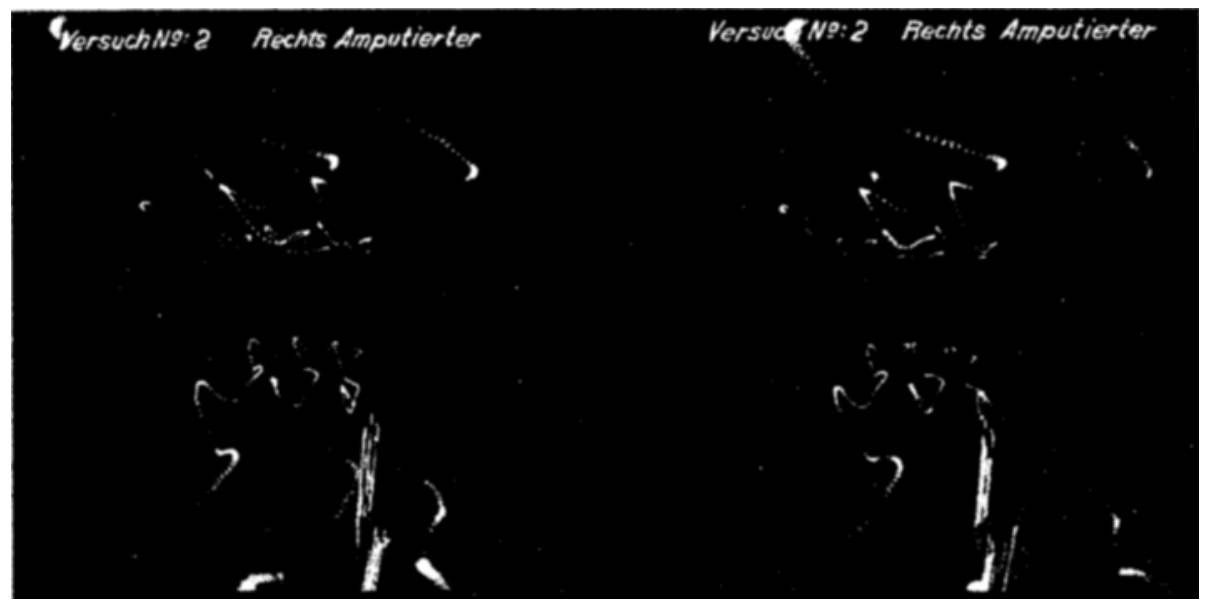

Abb. 14. Stereoskopaufnahme von hinten. 


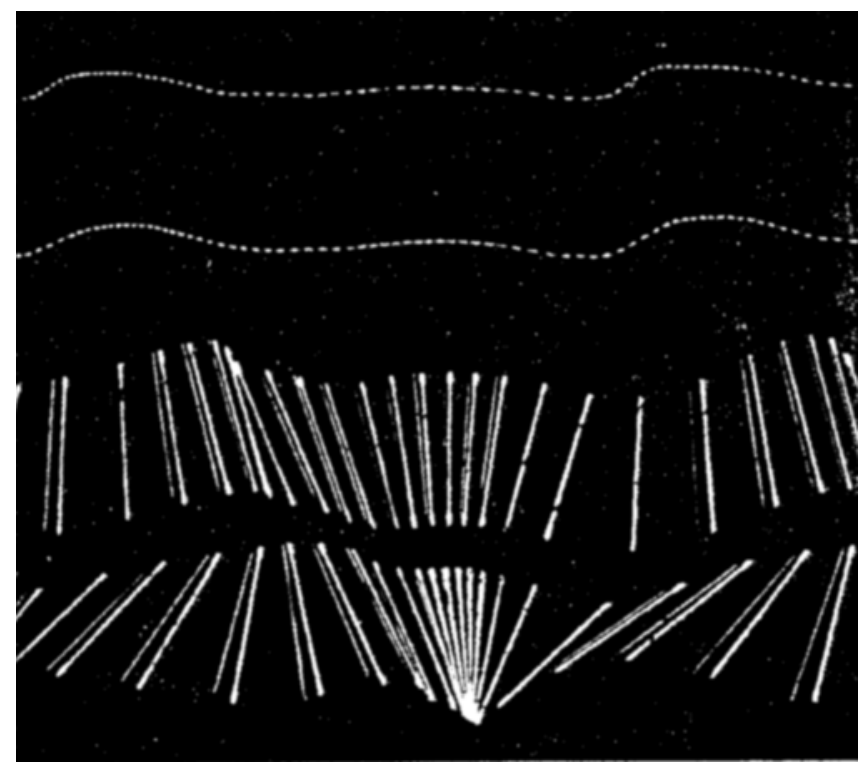

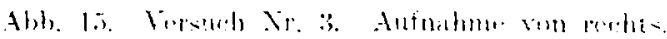

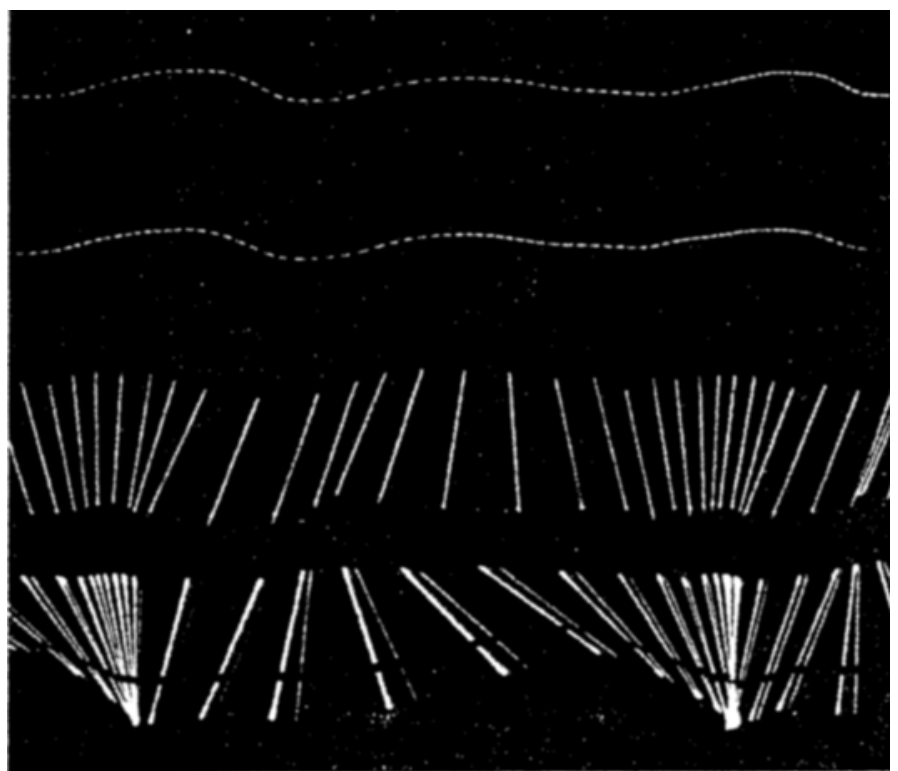

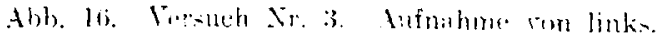




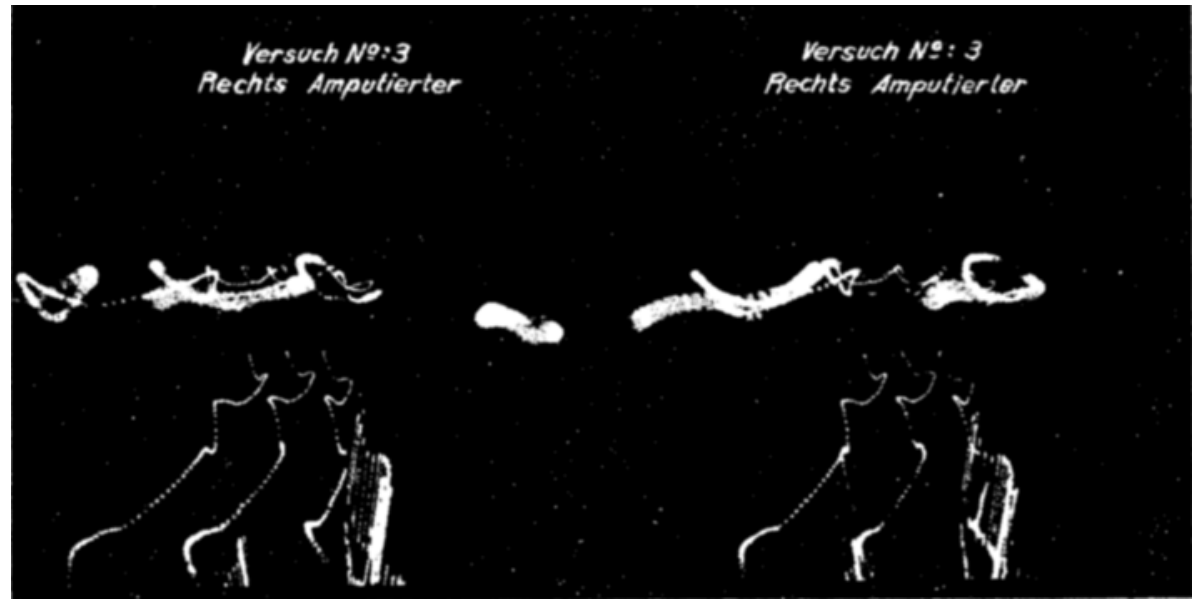

Abb. 17. Stereoskopaufnahme von hinten.

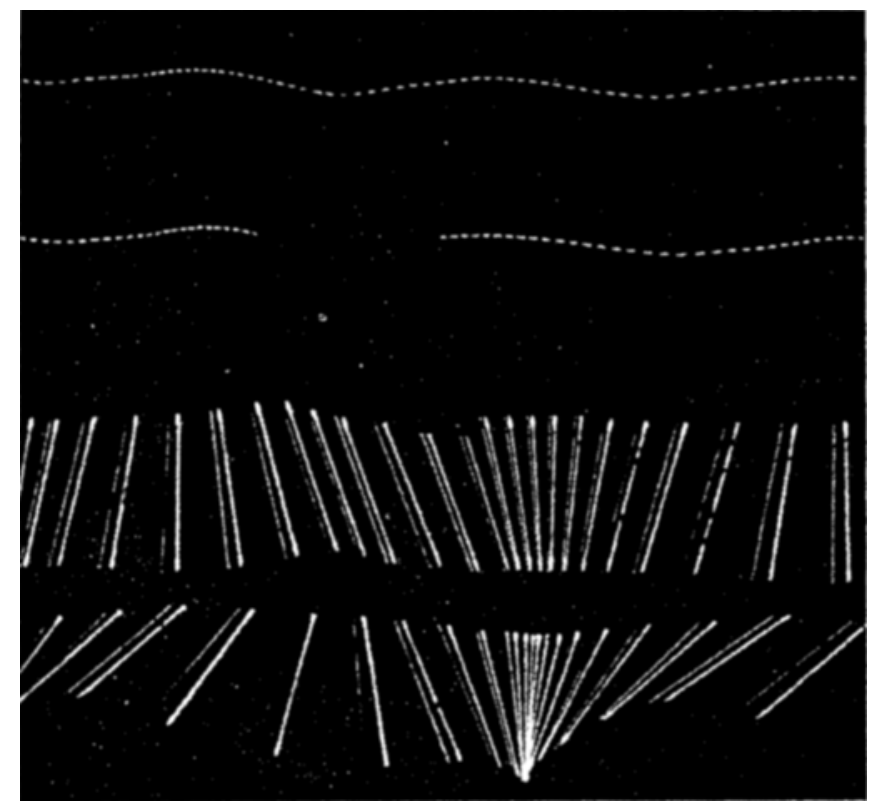

Abb. 18. Fersuch Nr. 4. Aufnahme ron rechts. 


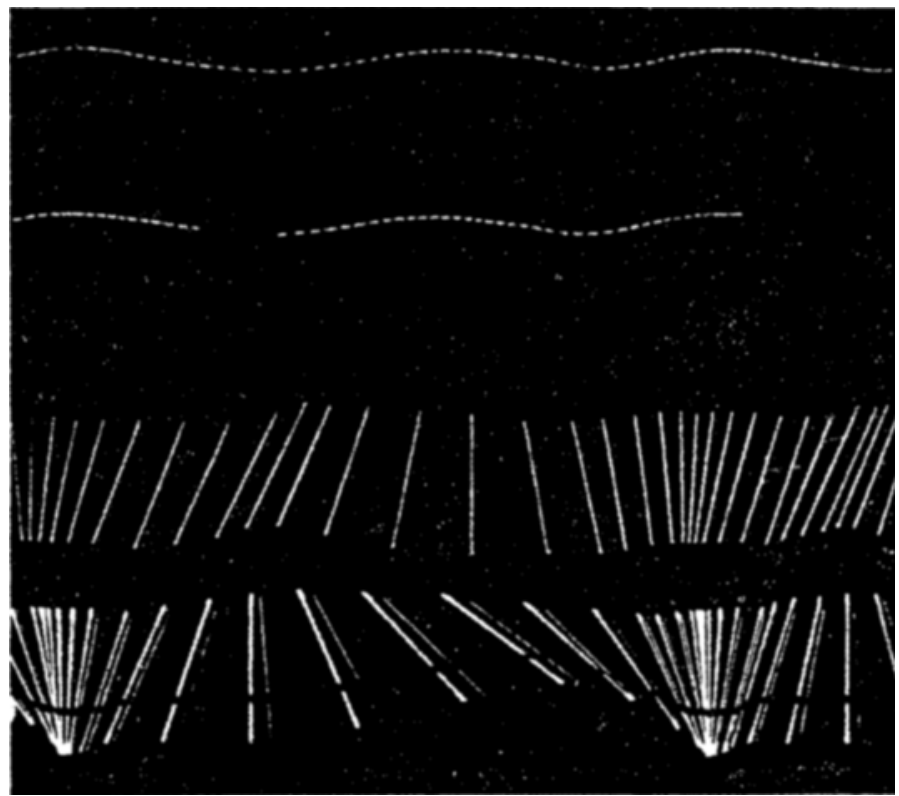

Abb. 14. Versuch Sir. t. Anfuithme von links.

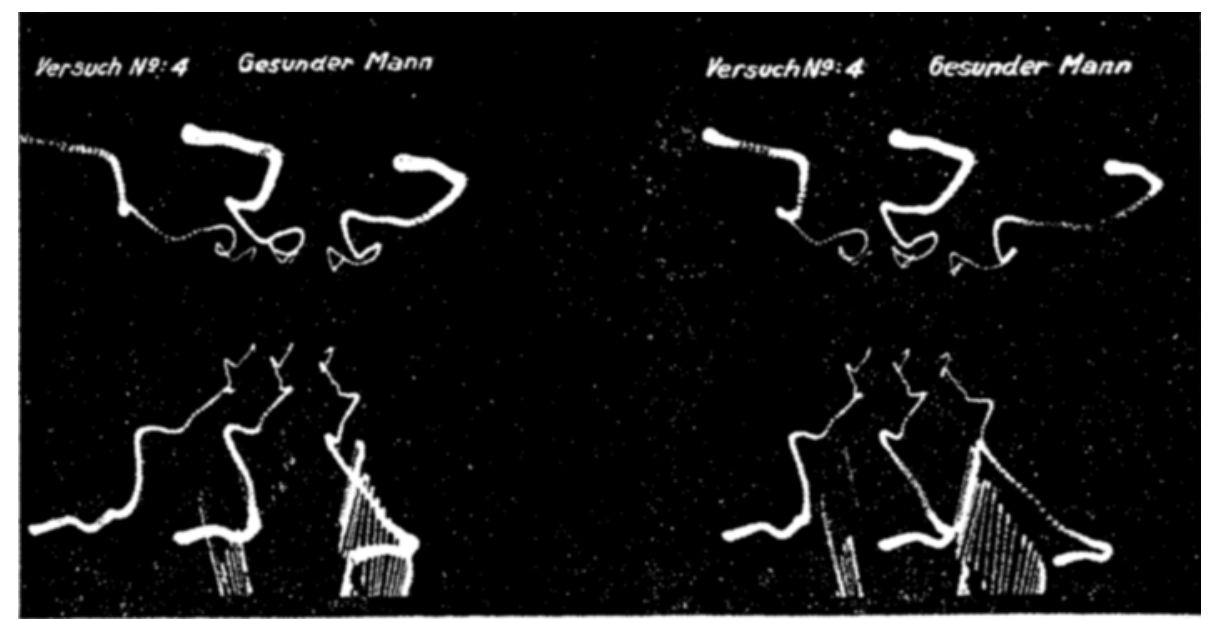

Abb. 20. Stereoskopaufnahme von hinten. 
Punkte müssen. so lange cine gleichmäßige Vorwürtsbewegnıng des Körpers stattfindet, da der Unterbrecher gleichmäßig arbeitete, die gleiche, der Ganggeschwindigkeit entsprechende Entfernung voneinander haben. Folgen die Punkte in der Gangrichtung in kürzeren Zwischenräumen aufeinander, so ist daraus zu erkennen, daß der betreffende Teil des Körpers sich langsamer als mit der Ganggeschwindigkeit vorbewegt, während bei weiterer Entfernung der Punkte ein Voreilen des betreffenden Teiles des Körpers festzustellen ist.

Projiziert man die Mitten der Punkte auf eine Wagerechte, so ergeben die auf der Wagerechten abgeschnittenen Stücke den in gleichen Zeiträumen, nämlich $1 / 35$ Sekunde zurückgelegten Weg. Zieht man dann von diesen Stücken
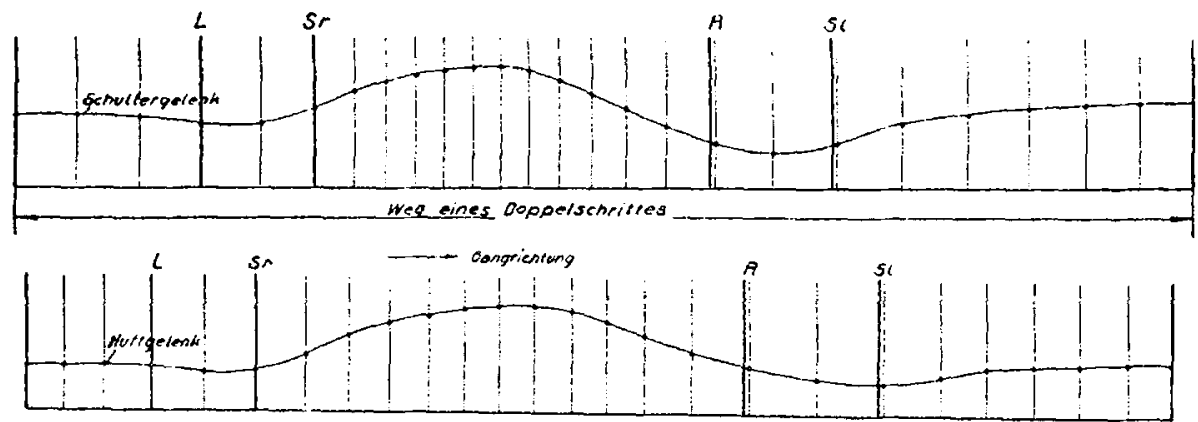

Abb. 21. Amputierter I. Amputierte (rechte) Seite.
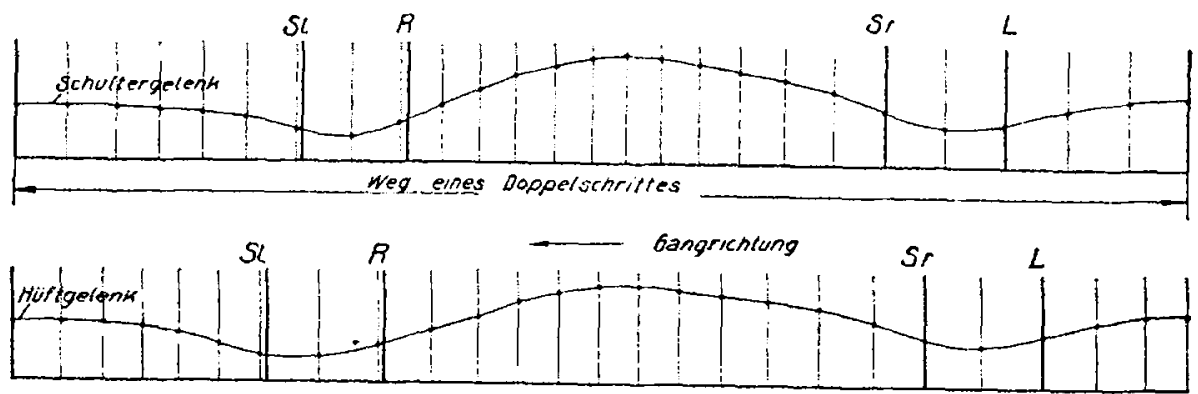

Abb. 22. Amputierter I. Gesunde (linke) Seite.

den in der gleichen Zeit bei mittlerer Geschwindigkeit zurückgelegten Weg $a b$, so ergibt sich das Maß der Voreilung oder des Zurückbleibens während 1/35 Sekunde in dem betreffenden Augenblick gegenïber der Stellung 7u Beginn dieses Zeitraumes. Um das der Abb. 2 entsprechende Diagramm fïr den Gang des Amputierten zu erhalten, muß man demnach zu gleichen auf der Zeit-(X-) Achse abgetragenen Abzissen Ordinaten auftragen, von denen jede folgende um das so gefundene $\mathrm{Ma} \beta$ größer bzw. kleiner ist als die vorhergehende. Der Anfangspunkt kann beliebig gewählt werden.

Die Konstruktion wurde für die Aufnahmen nach Abb. 9, 10 und Abb. 15, 16. also für beide untersuchten Individuen durchgeführt. Zur Erzielung größerer Genauigkeit wurden von den photographischen Platten zunächst vergrößerte positive Bilder hergestellt, und zwar wurde zur Vereinfachung der weiteren 
Konstruktion die Vergrößerung so gewählt, daß das positive Bild gerade ${ }^{1}{ }_{4}$ der natürlichen Große hatte. Sun wurde auf diesen Bildern je ein voller Doppelschritt ausgewählt. es ergaben sich dafür bei dem ersten Versuche (Abb. 9, 10) 50 Punkte. bei dem dritten Versuch (Abb. 15, 16) dagegen 52 Punkte. Dic Zeit eines Doppelschritts war also bei den beiden Amputierten um ${ }^{2 / 35}$ Sekunden verschieden. Der Deutlichkeit wegen wurde nur jeder zweite Punkt auf eine Wiugerechte projiziert, so daß die ganze Strecke eines Doppelsohrittes auf ihr in 2.5 bzw. 26 ungleiche Teile geteilt wurde (vgl. Abb. 21-24). Die Entfernung z. B. des $n$. Teilpunktes ron dem Anfangspunkt gibt demnach in diesen Ab-
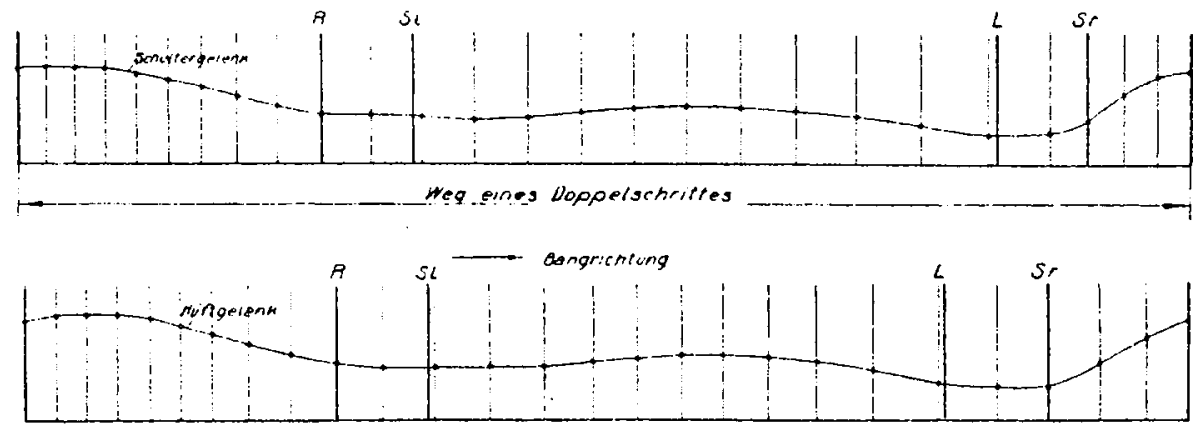

Abb. 23. Amputierter II. Amputierte (rechte) Steite.
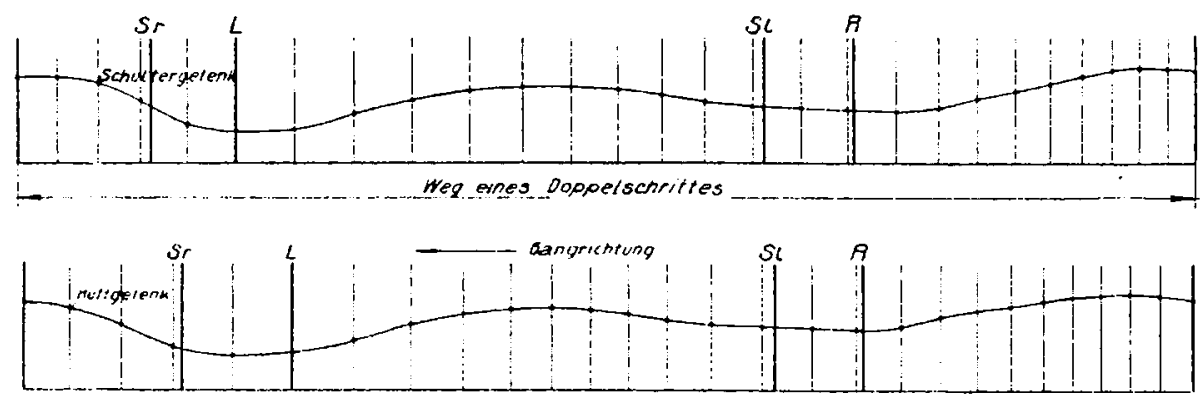

Abr). 24. Amputierter II. (resunde: (linke) Seite.

bildungen den zurïckgelegten Weg nach $2 . n$ Sekunden an. (Die Abbildungen sind verkleinerte Wiedergaben.)

Es wurde jetzt die ganze Strecke des Doppelschritts in 25 hzw. 26 . gleiche Teile geteilt; diese Teilpunkte entsprechen der Stellung eines mit gleichbleibender Geschwindigkeit vorbewegten Körpers, also etwa des Koordinatensystems, nach je ${ }^{3 / 35}$ Sekunden. Die Differenz zwischen den Entfernungen eines Teilpunktes der Abbildungen 21-24 rom Anfangspunkte und den Entfernungen des gleichnamigen Teilpunktes der gleichmäßigen Tejlung vom Anfangspunkte ergab nun, je nach dem ob sie ein positives oder negatives Vorzeichen hatte, die Strecke, um die die betreffende Lampe in dem dem Teilpunkte entsprechenden Augenblick vorgeeilt oder zurückgeblieben war. 
In Abb. 25 und 26 ist die Konstrutition für die rechte und linke Seite des Versuchs I (Amputierter I) fïr die Schultergelenk- und Hüftgelenkbewegung durchgeführt, in Abb. 27 und 28 für den Versuch III (Amputierter II).

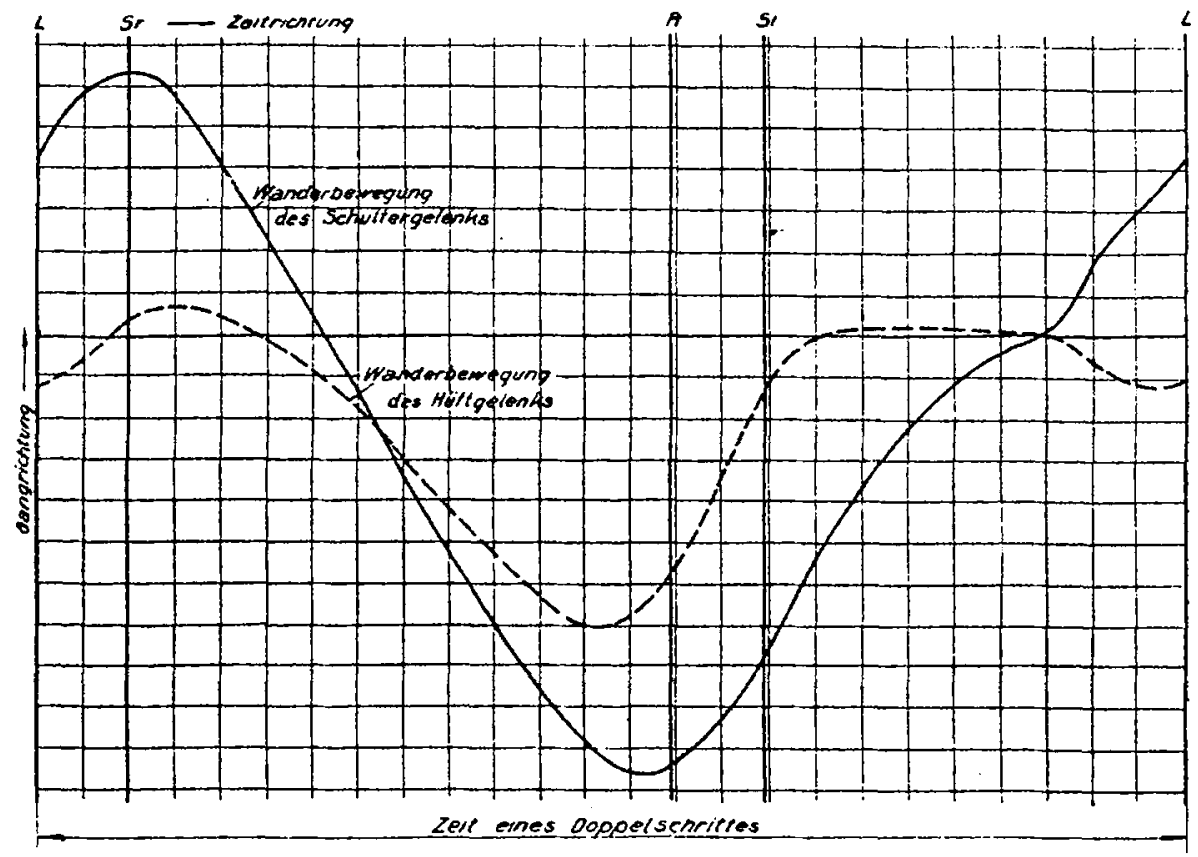

Abb. 25. Wanderbewegung des Amputierten I. Rechte (amputiorte) Stit:-

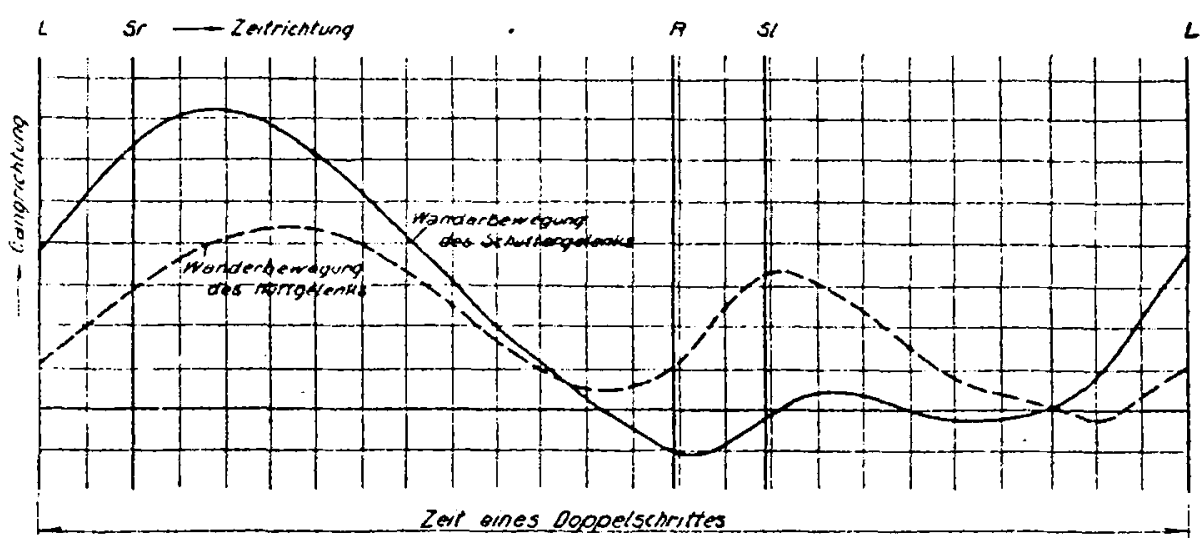

Abb. 26. Wanderbewegung des Amputierten I. Linke (gesunde) Stite.

Von ciner Konstruktion für den Versuch II wurde abgesehen, weil die Aufnahme derjenigen des Versuchs I außerordentlich ähnlich ist. Um die Bewegungen in der richtigen Größe zu erhalten, mußten die in der angegebenen Weise 
urhaltenen Differenzstrecken vierfach vergrößert werden, dla ja die veryrößerte Photographie nur $1 / 4$ natürlicher Größe hatte. Sie wurden dann als Ordinaten zu einer in 25 bzw. 26 gleiche Teile geteilten Zeitachse als Alszissenachse

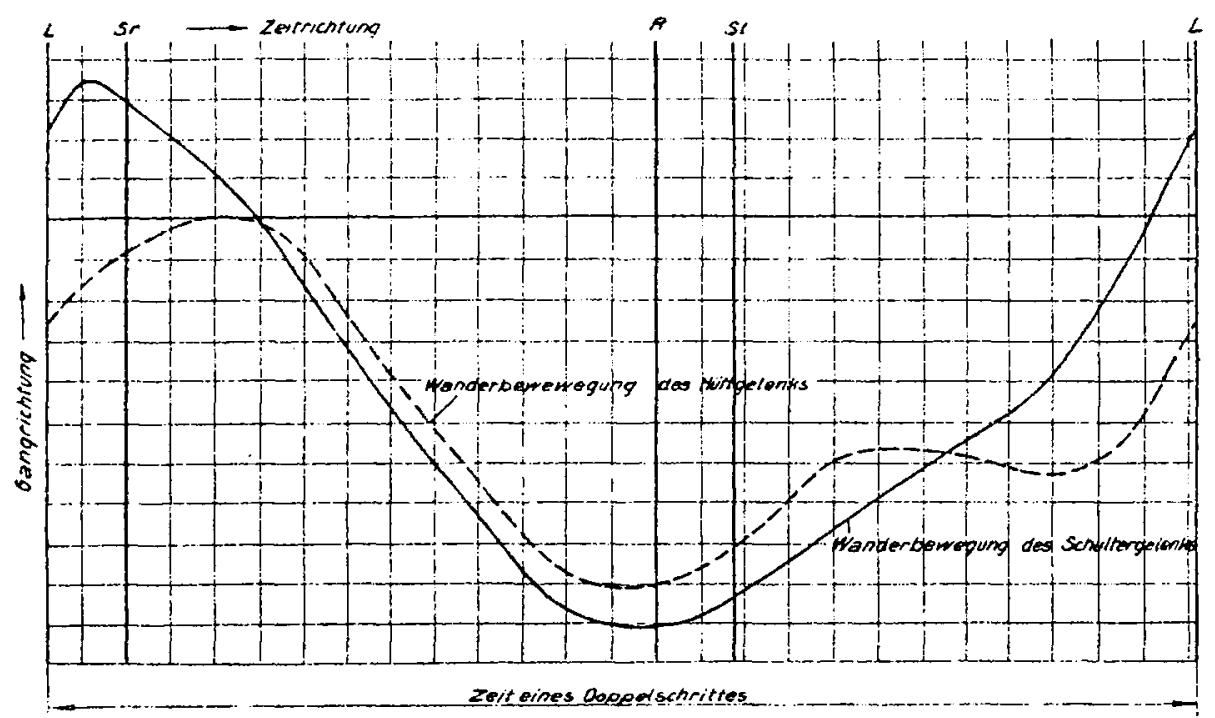

Abb. 27. Wanderbewegung des Amputierten II. Rechte (amputicrte') Seite.

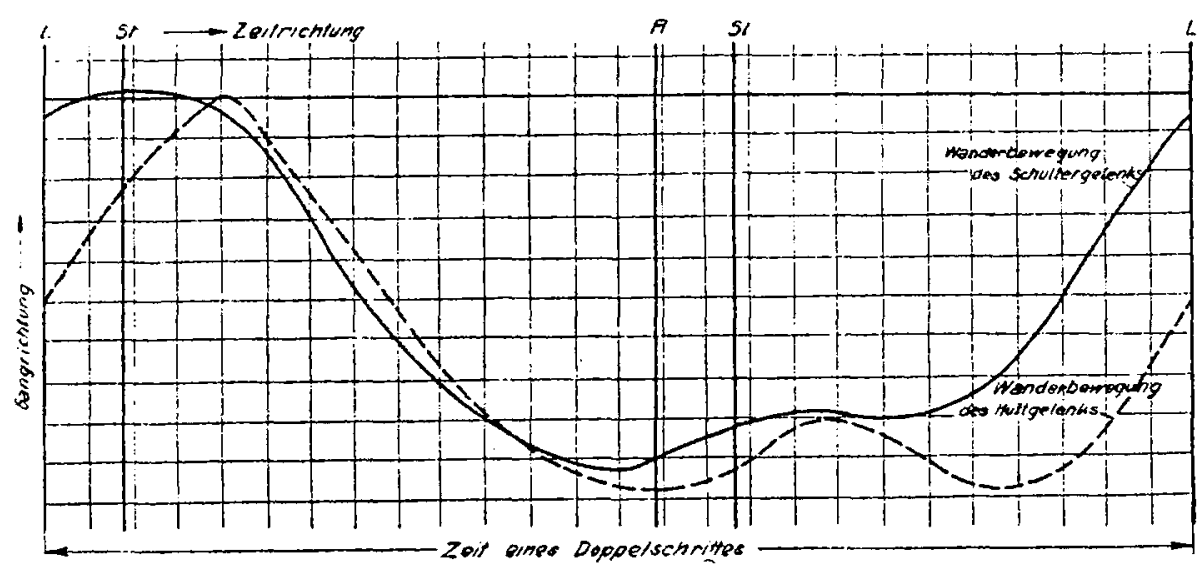

Abb. 28. Wanderbewegung des Amputierten IJ. Linke (gesunde) Seite.

aufgetragen ${ }^{1}$ ). Die O-Linie in diesen Diagrammen zeigt nicht die Mittelstellung, d. h. die normale aufrechte Haltung des Körpers, sondern die dem beliebig gewählten Zeitpunkte des Beginns des Doppelschritts entsprechende Körperlage; es kann demnach aus den Kurven nur der Charakter der Bewe-

1) Die Abbildungen 2:-58 sind verkleinert. Die Entfernung je zweier Teilstricht: stellt $1 \mathrm{~cm}$ dar. 
gung und der gesamte Ausschlag entnommen werden, nicht der Betrag der Bewegung aus der Mittellage nach vorn und nach rückwärts.

Unter Zuhilfenahme der Photographien konnten jetzt aus den Beinstellungen diejenigen Punkte ermittelt werden, an denen das Aufsetzen und Abschwingen der Beine stattfindet. Diese Stellen wurden durch die mit $R$, $\mathrm{I}_{2}, \mathrm{~S}_{\mathrm{r}}$ und $\mathrm{S}_{1}$ bezeichneten Tinien festgelegt.

\section{Y-Riehtung.}

Zur Feststellung der Bewegungs-Diagramme für die Y-Richtung müssen die Stereogramme (Abb. 1.1, 14 und 17) zu Hilfe genommen werden. Diese Bilder lassen, wie bereits erwähnt, wenn man sie durch das Stereoskop hetrachtet, nicht nur die Bewegung in der Y-Richtung erkennen, sondern zeigen don rollständigen Verlauf der Raumkurven. Man kann also für jeden Zeitpunkt den Ausschlag in der Y-Richting feststellen und gleichzeitig bestimmen, welchem Punkt der Bewegung in der X-oder besser der Z-Richtung dieser Zeitpunkt entspricht, worauf dann aus den Seitenaufnahmen die zu diesem Zeitpunkte gehörige Phase der Beinbewegung ermittelt werden kann. Infolge der Möglichkeit der Orientierung durch die Seitenphotogranme lïßt sich also der Verlauf der Y-Diagramme aus der Betrachtung der Bilder im Stereoskop zunächst dem Charakter nach aufzeichnen, die Größe der Bewegungen kann man aber nur durch den Vergleich mit dem unter gleichen Bedingungen aufgenommenen Stereoskopbild eines Gesunden abschützen, wobei man wiederum zur Gewinnung eines Maßstabes für die Größe der Bewegung des Gesunden r.twa die Fischerschen Kurven einsetzen müßte. Ein in dieser Weise konstruiertes Diagramm kann natürlich nicht sehr genau sein, man muß sich aber für die vorliegende Uintersuchung damit begnügen, wenn man die schwierigen und langwierigen photographischen Messingen vermeiden will.

\section{Z-Richtung.}

Die Diagramme für die Z-Richtung sind wiederum aus den Seitenphotogrammen zu entnehmen. Allerdings geben diese kein unmittelbares Bild der Bewegung in der Z-Richtung, da in ihnen die Hebungen und Senkungen der Rumpfgelenke als Ordinaten zu den in der betreffenden Zeit zurückgelegten Wegen nicht zu den Zeiten selbst als Abszissen erscheinen. Man muß deshalb die Hebungen und Senkungen in den Photogrammen in gleichen Zei tabständen also z. B. bei jedem zweiten Lichtpunkt messen und diese Strecken auf einer in gleiche Teile geteilten Zeitachse als Ordinaten abtragen. Dies ist für Versuch I und III in den Abb. 29-32 geschehen, und zwar wieder unter Benutzung der vergrößerten Photographien und nach Umrechung der ermittelten Strecken auf die natürliche Größe. Jede Abbildung enthält die Hebbewegung je eines Sohultergelenkes und des dazu gehörigen Hüftgelenkes.

Um festzustellen, ob die angegebene Methode der Konstruktion ähnliche Resultate ergibt wie die umständliche Rechnungsmethode, die Fischer anwandte, wurden auch für den Gesunden (Versuch IV) die Diagramme der Winderbewegung und der Hebbewegung in der beschriebenen Weise konstruiert. 
Die Rumpfbewegung der Kunstbeintrïger und ihr Zusammenhang usw.

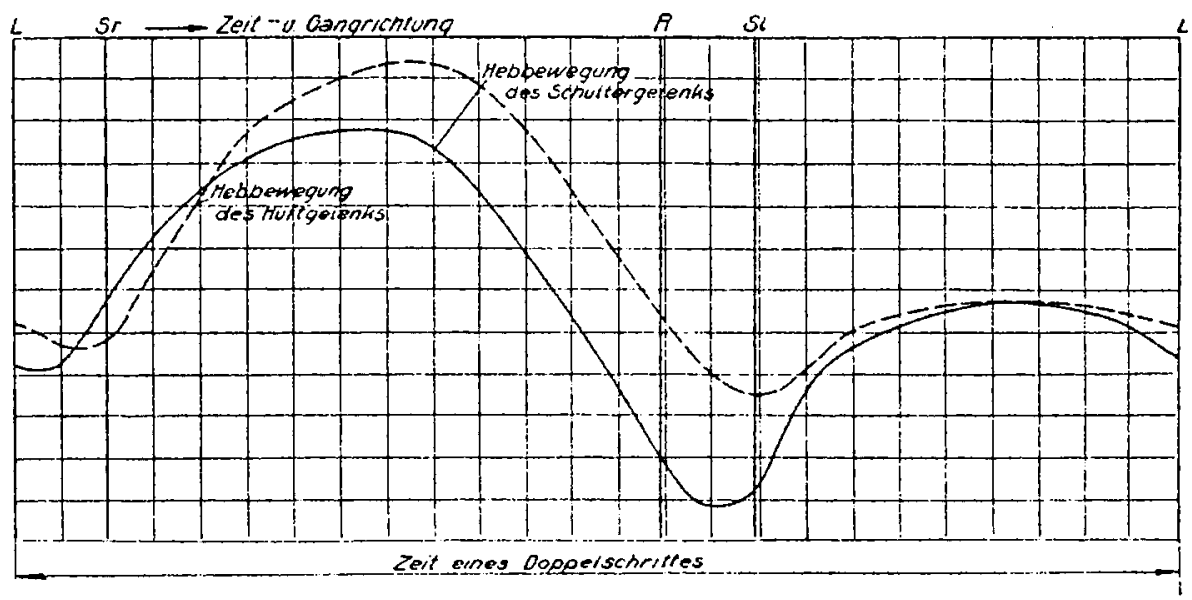

Abb. 29. Hebbewegung des Amputierten I. Rechte (amputicrte) Seite.

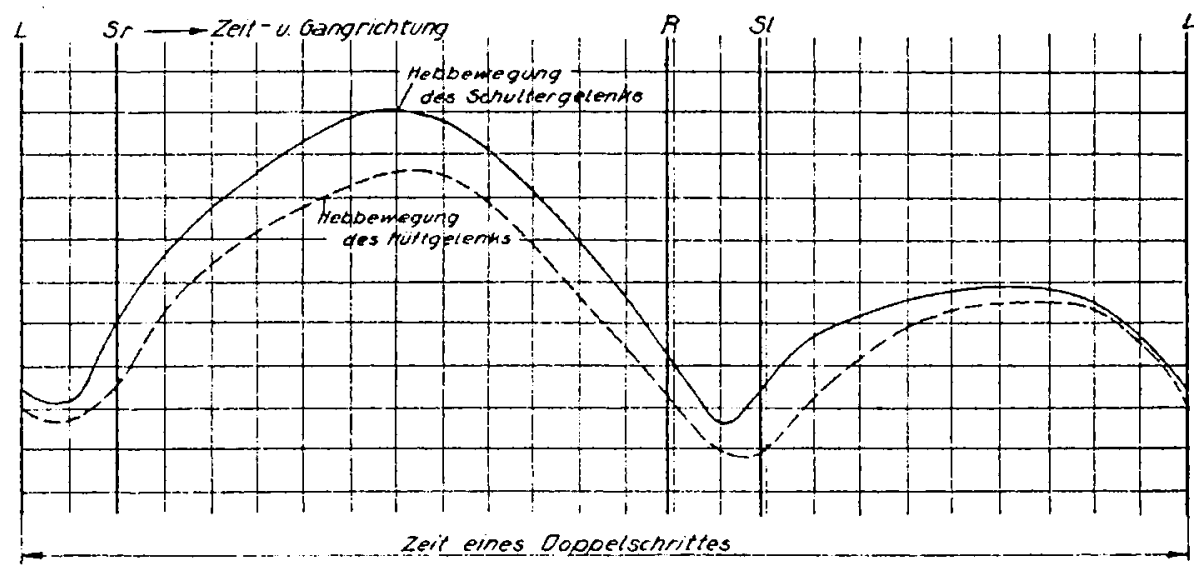

Alb. 30. Hebbewegung des Amputierten I. Linke (gesunde) Sejte.

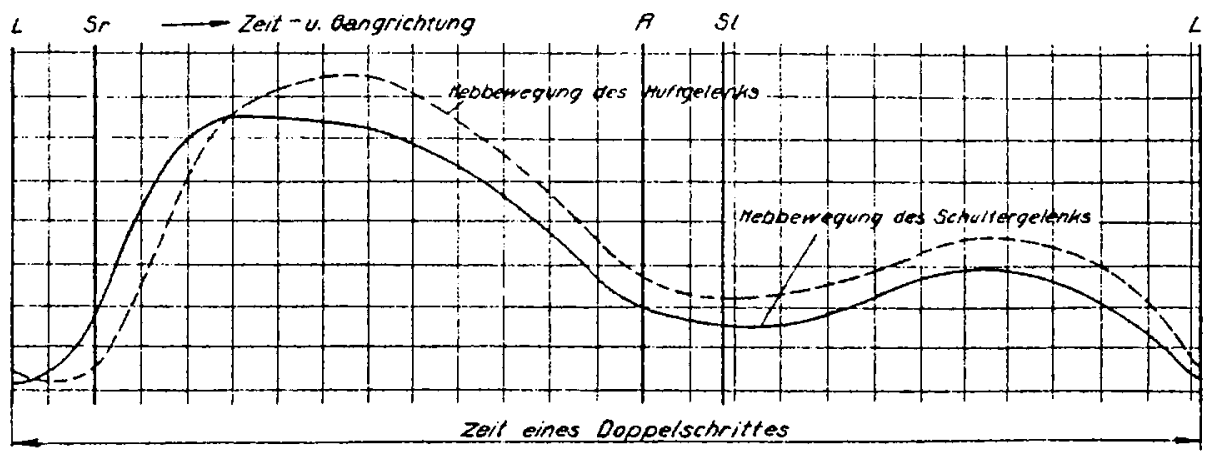

Abb. 31. Hebbeweging des Amputierten II. Rechte (amputierte) Seite. 
Abb. 33 und 34 zeigen je einen Doppelschritt aus den vergrößerten Seitenphotogrammen, wobei wieder ron jedem zweiten Lichtpunkt aus cin Lot auf eine darunter gezogene Wagerechte gefillt ist. Die Kurren für die Wander-

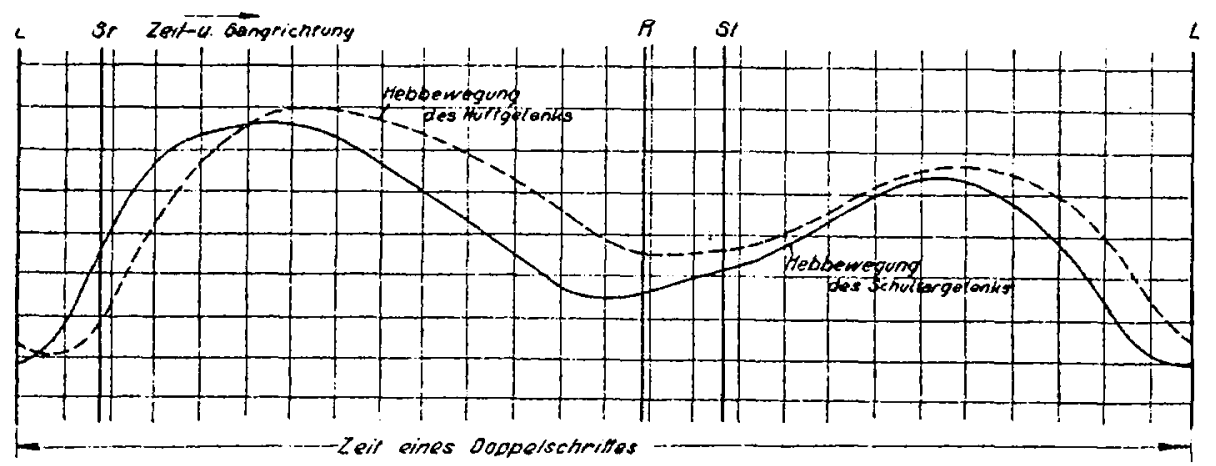

Abb. 32. Hebbewegung des Amputierten IL. Linke (gesunde) Seite.
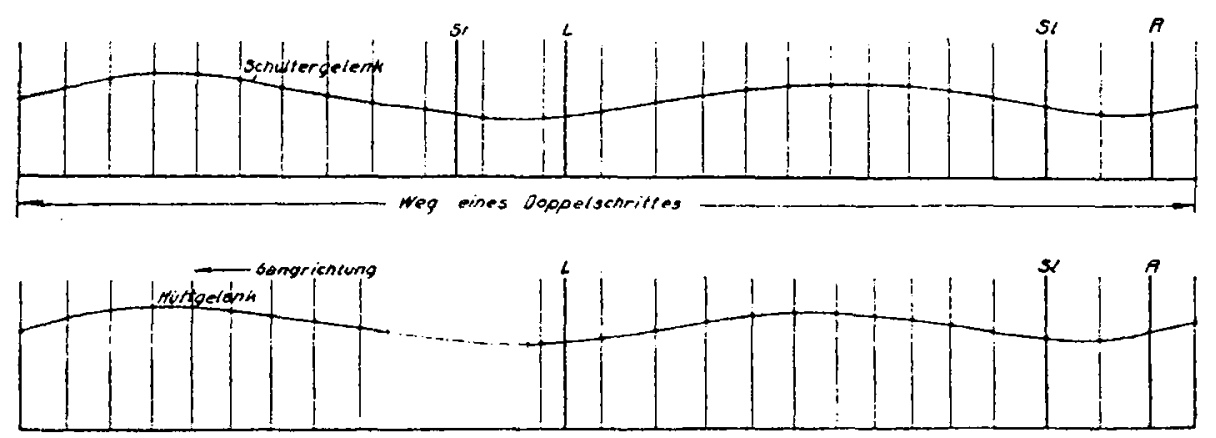

Abb. 33. Gesunder Mann. Linke Seite.

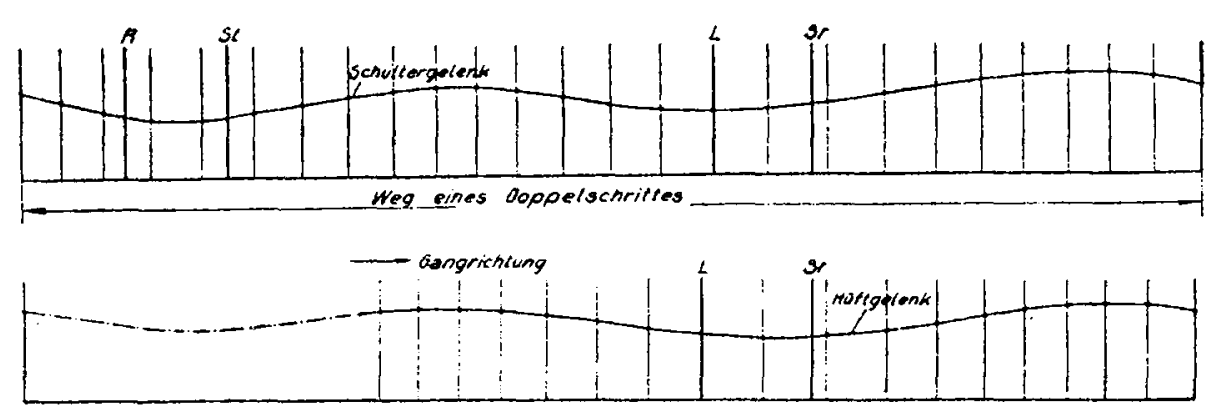

1bb. 34. Gesunder Mann. Rechte Seite.

bewegung der Schultergelenke sind in Abb. 35, der Hüftgelenke in Abb. 36 clargestellt; gleichzeitig enthalten diese Abbildungen die nach clen Fischersehen Zahlen konstruierten Kurven (Abb. 2) in punktierten Linien. 
Berücksichtigt man, daß die beim Gesunden verhältnismäßig geringen Winderbewegungen der Gelenke sehr von äußeren Zufälligkeiten abhängig sein können, z. B. kleinen Hindernissen, auch Angewohnheiten des Individuums. die die Form der Kurven in einzelnen beeinflussen, so wird man die Kurven des Versuches IV als gut übereinstimmend mit den Fischerschen Kurven bezeichnen können. Maxima und Winima liegen in beiden Fällen etwa bei der gleichen Phase des Doppelschrittes. In dieser Bezichung stimmen beim Schultergelenk die beiden Teile des Doppelschritts bei Versuch IF noch besser

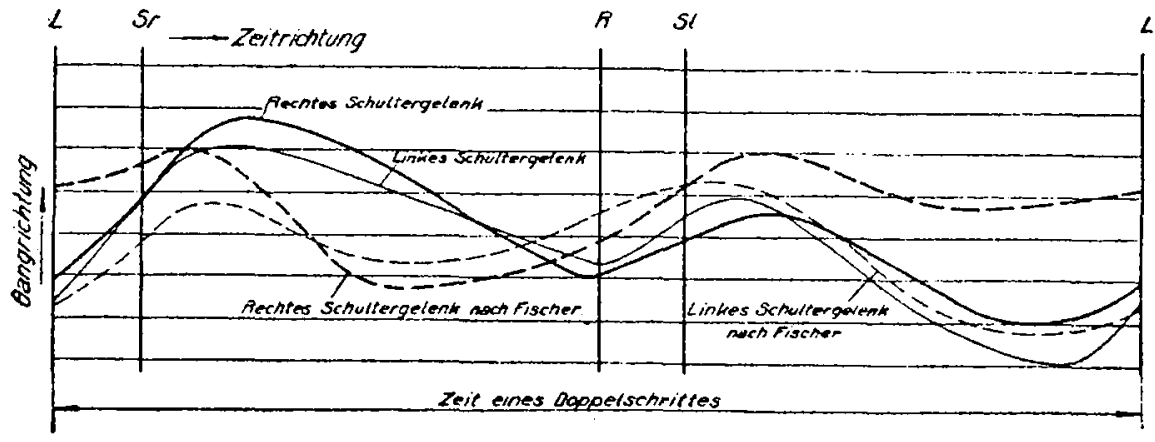

Abb. 33. Wanderbewegung der Schultergelente beim Gesunden. (Versuch 4 ).

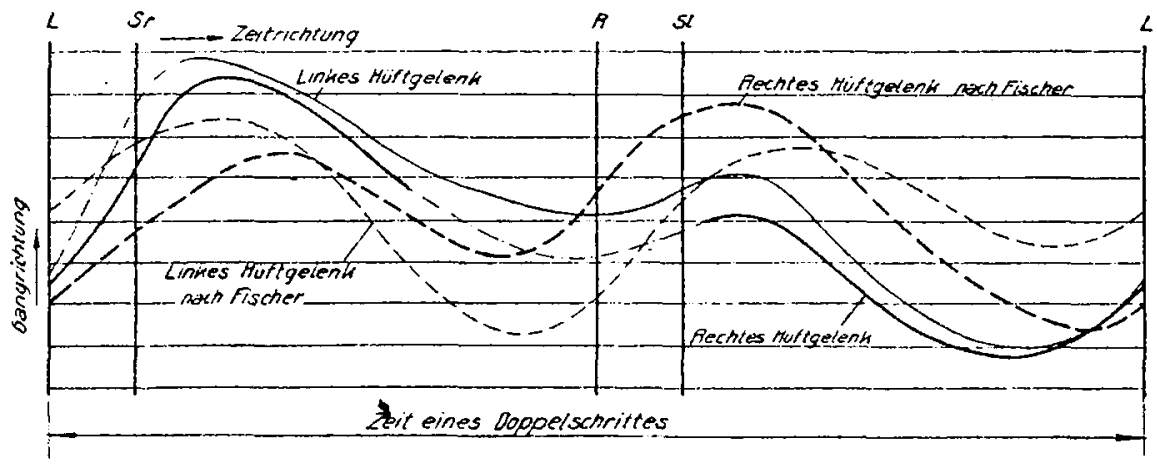

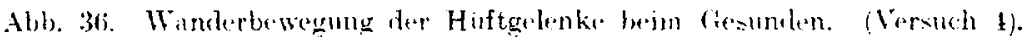

überein als bei dem Fischerschen Versuch. Das bei dem Fischerschen Versuch vorhandene unnormale Verhalten des rechten Schultergelenks ist nicht zu bemerken, dagegen zeigt sich bei Versuch IT während des Schwingens des rechten Beines ein größerer Ausschlag als beim Schwingen des linken Beines. Der Gesamtausschlag ist bei beiden Versuchen insbesondere beim Hüftgelenk nur wenig verschieden, auch kann man bei Versuch $\Pi$ die gleichen Drehbewegungen der Schulter- und Hüftlinien nenigstens der Art nach erkerinen. Mit anderen Worten: Die beiden Versuche ergeben Kurven gleichen Charakters, deren einzelne Unterschiede wohl auf das individuell verschiedene Verhalten der beiden Versuchsindividuen zurückzuführen ist. 
Auch die Hebbewegungen, die für die Schultergelenke in Abb. 37 und für die Hüftgelenke in Abb. 38 dargestellt sind, stimmen bei beiden Versuchen gut überein. Maxima und Minima decken sich genau, der Gesamtausschlag ist in beiden Fälen ziemlich der gleiche und auch die nur geringen Drehbewegungen der Hüft- und Schulterlinie in der senkrechten Ebene sind bei beiden Versuchen sehr ahnlich.

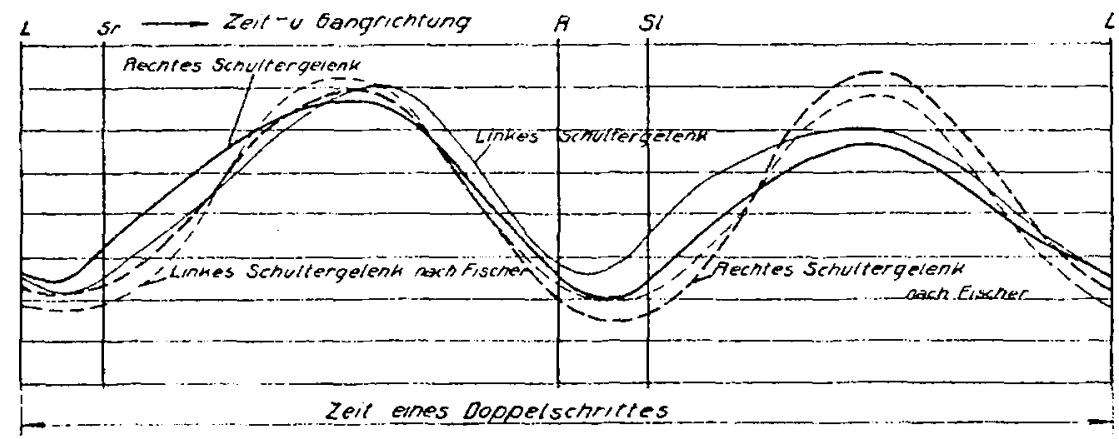

Abb. 37. Heblewegrung der Schultergelenke bein Gesunden. (Versueh 4).

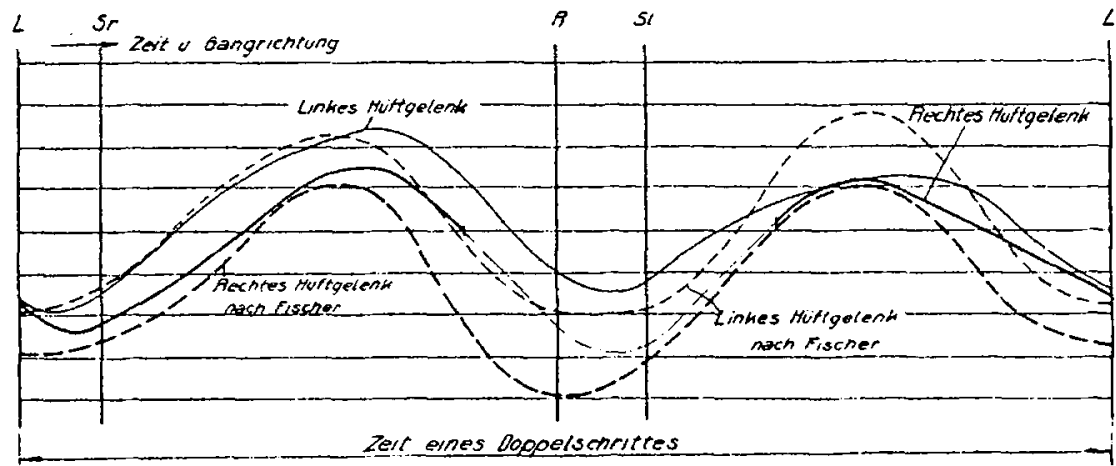

Abb. 38. Hebbewegung der Hüftgelenke beim Gesunden. (Versuch 4).

E⿺ ist aus dem Vergleiche zu ersehen, daß in allen Fällen, in denen es sich nur darum handelt, den Charakter der Rumpfbewegungen beim Gange und ihre Gesamtausschläge zu studieren, das angewandte Verfahren hinreichend genaue Resultate ergeben dürfte; es wird daher bei der nun vorzunehmenden Betrachtung der Rumpfbewegung des Kunstbeintrïgers ausreichen, da ja nur die prinzipiellen Unterschiede zwisehen dieser Bewegung und der Rumpfbewegung des Gesunden und ihr Zusammenhang mit der konstruktiven Ausbildung von Kunstbeinen überhaupt behandelt werden soll. 